\title{
Tachyons in throat cosmology
}

\section{S. Halter, ${ }^{a}$ B.v. Harling ${ }^{b}$ and A. Hebecker ${ }^{c}$}

${ }^{a}$ Max-Planck-Institut für Physik (Werner-Heisenberg-Institut), Föhringer Ring 6, D-80805 München, Germany

${ }^{b}$ School of Physics, University of Melbourne, Victoria 3010, Australia

${ }^{c}$ Institut für Theoretische Physik, Universität Heidelberg, Philosophenweg 16 und 19, D-69120 Heidelberg, Germany

E-mail: halter@mppmu.mpg.de, bvo@unimelb.edu.au, a.hebecker@thphys . uni-heidelberg.de

ABSTRACT: Tachyonic 5d scalars are generically present in Randall-Sundrum-like models. In particular, they are known to be part of the 5 d effective description of the KlebanovStrassler throat. When moving from the IR to the UV region, the $5 \mathrm{~d}$ bulk profile of Kaluza-Klein excitations of tachyons decays more slowly than that of massless scalars or the graviton. As a result, tachyons in many cases dominate the coupling between IR- and UV-localized sectors, leading to a very significant enhancement of energy-transfer or decay rates from the IR to the UV. This can dramatically affect the reheating of the Standard Model after brane inflation and the decay of throat dark matter.

KEYwords: Flux compactifications, Cosmology of Theories beyond the SM

ARXIV EPRINT: 0912.2111 


\section{Contents}

1 Introduction $\quad 1$

2 Tachyons in a slice of $\mathrm{AdS}_{5} \quad 3$

3 Decays mediated by $\mathrm{AdS}_{5}$ fields $\quad 6$

3.1 Generic UV-brane mass term 6

3.2 Tuned UV-brane mass term 9

4 Situations dominated by the tachyon $\quad 11$

$\begin{array}{lll}5 & \text { CFT interpretation } & 12\end{array}$

6 Application to throats in flux compactifications 16

$\begin{array}{lll}7 & \text { Cosmology } & 21\end{array}$

8 Conclusions 24

$\begin{array}{ll}\text { A Decay rates with Bessel functions } & 27\end{array}$

\section{Introduction}

Strongly warped regions or 'throats' are a generic feature of type IIB flux compactifications [1-6]. It can even be argued that, in certain regions of the 'landscape', they are statistically unavoidable [7]. The prime example is the Klebanov-Strassler (KS) throat (or warped deformed conifold) [8]. It can be viewed as a stringy version of a Goldberger-Wisestabilized Randall-Sundrum (RS) model [9] and is thus phenomenologically interesting for all the well-known reasons making the RS model so attractive. Moreover, throats play a key role in mechanisms realizing de Sitter vacua [10] and in string-theoretic models of inflation, such as brane-antibrane inflation [11].

In warped compactifications, couplings between fields localized in the UV and IR regions (regions of weak and strong warping, respectively) are generically suppressed by powers of the warp factor $[12,13]$. In cosmology, two implications of this suppression are of particular interest: ${ }^{1}$ One is the long lifetime of certain IR-localized modes, which can hence play the role of 'throat dark matter' [14-19]. The other is the small energy transfer rate from a plasma of such modes (created at the end of warped brane inflation) to any

\footnotetext{
${ }^{1}$ In non-cosmological applications of warped geometries, such as supersymmetry breaking and flavor model building, related effects are known as 'sequestering' (see e.g. [25-29]). The technical questions arising in this context are somewhat different and we will not address them in the following.
} 
UV-localized sector [20-24]. This can cause problems for the reheating of the Standard model after inflation in a throat.

The main point of the present paper is to demonstrate that tachyonic $5 \mathrm{~d}$ scalars can play a central role in coupling IR- and UV-localized sectors and to discuss some of the immediate cosmological implications.

The importance of tachyons in this context is easily understood: Recall first that the presence of tachyons is $\mathrm{AdS}_{5}$ is natural from the perspective of $5 \mathrm{~d}$ supersymmetry [30, 31] and observed in concrete examples, such as the compactification of type IIB supergravity to $\mathrm{AdS}_{5}$ on $T^{1,1}$ [32-34]. It is well-known that tachyons do not destabilize infinite $\mathrm{AdS}_{5}$ as long as their negative mass-squared respects the Breitenlohner-Freedman bound [35, 36]. On a slice of $\mathrm{AdS}_{5}$, tachyons generically introduce an instability. In supersymmetric models on a slice of $\mathrm{AdS}_{5}$, stability is maintained by a positive mass-squared operator localized at the UV brane [31]. Such a brane-mass term can also be used to ensure the stability of nonsupersymmetric Randall-Sundrum models with tachyons [37-39]. ${ }^{2}$ Now, for any massive 5d scalar, its efficiency in mediating interactions between IR and UV brane is determined by the $5 \mathrm{~d}$ bulk profile of its lowest Kaluza-Klein modes. Generically, the value of IR-localized modes is exponentially suppressed near the UV brane. This exponential suppression is governed by the $5 \mathrm{~d}$ mass-squared. Thus, it is natural to expect that tachyonic scalars will provide the strongest coupling between the UV and IR brane. In the following, we study this general idea at the quantitative level.

Our main point can be made in a simple 5d toy model: a single tachyonic scalar on a slice of $\mathrm{AdS}_{5}$. Any of its low-lying IR-localized KK modes can be taken to represent a typical particle of the IR sector. The UV sector to which such a KK mode might decay is modeled as a $4 \mathrm{~d}$ gauge theory localized on the UV brane. Our $5 \mathrm{~d}$ scalar couples to the $F^{2}$ term of this theory through its value at the UV-brane. We calculate the corresponding decay rate and find it to be of the same order of magnitude as that resulting from the mediation of the graviton. ${ }^{3}$ This result arises from two compensating effects: On the one hand, the modes of a tachyon decay more slowly than those of a graviton when moving from the IR to the UV. On the other hand, they are subject to an extra suppression due to the UV-localized mass term (which is necessary for stability).

From the above, it is clear that in many relevant situations the tachyon is, in fact, bound to dominate: Namely, massless 5 d scalars are expected to obtain a large UV mass term in generic, fully stabilized models. The $5 \mathrm{~d}$ graviton, which has the profile of a massless scalar and no UV mass term, can not mediate the decay of spin-zero states. Thus, the tachyon dominates the decay of low-lying IR-localized spin-zero states or 'glueballs'. We illustrate this in a simplified KKLMMT-type setup for warped reheating. It turns out that tachyons dominate the energy transfer to the Standard Model. They can thus help to avoid the complete dissipation of inflationary energy density into gravitons, thereby solving a generic problem of (warped) brane inflation.

\footnotetext{
${ }^{2}$ Alternatively, one can impose the Dirichlet boundary condition at the UV brane [40]. This corresponds to the limit of sending the boundary mass to infinity.

${ }^{3}$ The bulk profile of modes of the $5 \mathrm{~d}$ graviton is the same as that of a massless $5 \mathrm{~d}$ scalar.
} 
Our paper is organized as follows: section 2 provides a pedagogical introduction to tachyons in a slice of $\mathrm{AdS}_{5}$. Building on this discussion, section 3 gives a simple and intuitive derivation of the decay rate of the IR-localized modes of a $5 \mathrm{~d}$ tachyon to a UVlocalized sector. It is followed by a discussion of situations in which the tachyon dominates the decay of IR-localized states in section 4. In section 5, we explain our results from the perspective of the dual CFT, finding further support for our intuitive picture and our calculations. We then discuss the applicability of our $5 \mathrm{~d}$ analysis to presumably more realistic and general throat geometries in section 6 . Finally, in section 7 , we turn to cosmological applications, in particular to effects on reheating and dark matter decay. Our Conclusions are followed by an appendix in which we provide a Bessel function analysis supporting our order-of-magnitude calculation in the main text.

Cosmological implications of tachyonic 5d scalars in a warped throat were discussed in [15] and, more recently, in [19]. The latter analysis includes processes which, in our language, can be interpreted as tachyon-mediated decays from the IR- to the UV-localized sector. We discuss the relation of [19] to our work in section 6.

\section{Tachyons in a slice of $\mathrm{AdS}_{5}$}

To set up our notation, we recall that an RS I model [41] is defined by a slice of $\mathrm{AdS}_{5}$ with metric

$$
d s^{2}=e^{-2 k y}\left(\eta_{\mu \nu} d x^{\mu} d x^{\nu}\right)+d y^{2},
$$

where $k=1 / R$ is the inverse curvature radius. The slice is bounded by two 3 -branes at $y_{\mathrm{UV}}=0$ and $y_{\mathrm{IR}}=\ell$. In the following, we will focus on the dynamics of a scalar $\Phi$ with action

$$
S_{5 d}=\int d^{4} x d y \sqrt{-G} \frac{1}{2} \Phi\left[\nabla^{2}-M^{2}\right] \Phi,
$$

where $\nabla^{2}=(\sqrt{-G})^{-1} \partial_{M}\left(\sqrt{-G} G^{M N} \partial_{N}\right)$ and $G_{M N}$ is the $\mathrm{AdS}_{5}$ metric. Capital indices run over $\{0, \ldots, 3\}$ and $y$. We assume that the model is already stabilized, e.g. via the Goldberger-Wise mechanism [42], and that $\Phi$ is an additional scalar field, not related to the stabilization.

It will be convenient to use the coordinate $z=k^{-1} e^{k y}$ and the corresponding conformally flat metric

$$
d s^{2}=\frac{1}{(k z)^{2}}\left(\eta_{\mu \nu} d x^{\mu} d x^{\nu}+d z^{2}\right) .
$$

Expanding $\Phi$ in KK modes,

$$
\Phi(x, z)=\sum_{n} \varphi_{n}(z) \chi_{n}(x)
$$

we have a set of canonically normalized $4 \mathrm{~d}$ scalars $\chi_{n}$ with masses $m_{n}$, the wave functions of which satisfy the equations

$$
\left(z^{2} \partial_{z}^{2}-3 z \partial_{z}+m_{n}^{2} z^{2}-\frac{M^{2}}{k^{2}}\right) \varphi_{n}(z)=0
$$


To specify boundary conditions, it is convenient to view the extra dimension as an $S^{1} / \mathbb{Z}_{2}$ orbifold. Thus, our $\varphi_{n}$ are defined on the double cover of the interval $\left[z_{\mathrm{UV}}, z_{\mathrm{IR}}\right]$, where $z_{\mathrm{UV}}=k^{-1}$ and $z_{\mathrm{IR}}=k^{-1} e^{k \ell}$. We assume that $\Phi$ is even under the $\mathbb{Z}_{2}$ symmetry transformation, implying that $\varphi_{n}$ is symmetric under $\left(z-z_{\mathrm{UV}}\right) \rightarrow-\left(z-z_{\mathrm{UV}}\right)$ and periodic with period $2\left(z_{\mathrm{IR}}-z_{\mathrm{UV}}\right)$.

In terms of the rescaled field

$$
\psi_{n}(z) \equiv(z k)^{-3 / 2} \varphi_{n}(z)
$$

Equation (2.5) takes the form of a 1-dimensional Schrödinger equation [41]

$$
\left(-\partial_{z}^{2}+V(z)\right) \psi_{n}(z)=m_{n}^{2} \psi_{n}(z)
$$

with 'energy' $m_{n}^{2}$ and potential

$$
V(z)=\frac{\alpha^{2}-\frac{1}{4}}{z^{2}}-\frac{3}{z_{\mathrm{UV}}} \delta\left(z-z_{\mathrm{UV}}\right)+\frac{3}{z_{\mathrm{IR}}} \delta\left(z-z_{\mathrm{IR}}\right) .
$$

Here $\alpha \equiv \sqrt{4+M^{2} / k^{2}}$, and the $\delta$-function contributions come from the rescaling in the presence of boundary conditions. The solutions of eq. (2.7) are Bessel functions of order $\alpha$ (cf. appendix A) and all that follows could be derived from a careful analysis of the behaviour of these functions. However, we find it more illuminating to make our main qualitative points using a parametric analysis of approximate but explicit solutions.

According to eqs. (2.7) and (2.8), we are basically looking for the solutions of a quantum mechanical problem on an interval. ${ }^{4}$ The coefficient of the $1 / z^{2}$ term in the potential is subject to the Breitenlohner-Freedman bound [35, 36] $M^{2} \geq-4 k^{2}$ (corresponding to $\alpha \geq 0$ ), which ensures the stability of the $\mathrm{AdS}_{5}$ vacuum. Figures 1 and 2 show the potential for the particularly interesting cases $M=0$ and $M=-4 k^{2}$. Standard KK-mode intuition tells us that all $m_{n}^{2}$ are positive for $M^{2}>0$, while $m_{0}=0$ for $M=0$. We then clearly expect the presence of negative 'energy eigenvalues' $m_{n}^{2}$ for 'tachyonic' $M^{2}<0$. This is not tolerable from the perspective of the $4 \mathrm{~d}$ effective field theory. Indeed, while the Breitenlohner-Freedman bound is sufficient to ensure the stability of infinite AdS space, it does not guarantee the stability of a slice of AdS.

One way to avoid this instability is to introduce a UV-brane-localized mass operator [37-39]. We therefore supplement eq. (2.2) with

$$
S_{\mathrm{UV}}=-\int d^{4} x d y \sqrt{-G} \lambda k \Phi^{2}(x, y) \delta\left(y-y_{\mathrm{UV}}\right),
$$

where we have written the dimensionful coefficient as a product of $k$ and a dimensionless parameter $\lambda \geq 0$. This mass term modifies the potential,

$$
V(z)=\frac{\alpha^{2}-\frac{1}{4}}{z^{2}}+\frac{2 \lambda-3}{z_{\mathrm{UV}}} \delta\left(z-z_{\mathrm{UV}}\right)+\frac{3}{z_{\mathrm{IR}}} \delta\left(z-z_{\mathrm{IR}}\right),
$$

\footnotetext{
${ }^{4}$ More precisely, we are looking for the even solutions on the $S^{1}$ covering space of that interval.
} 


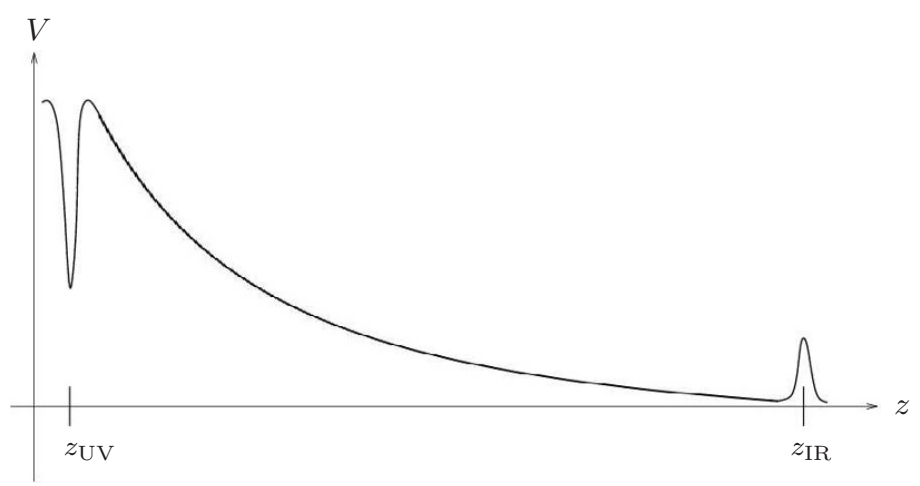

Figure 1. Potential in the effective Schrödinger equation for a $5 \mathrm{~d}$ mass $M=0$.

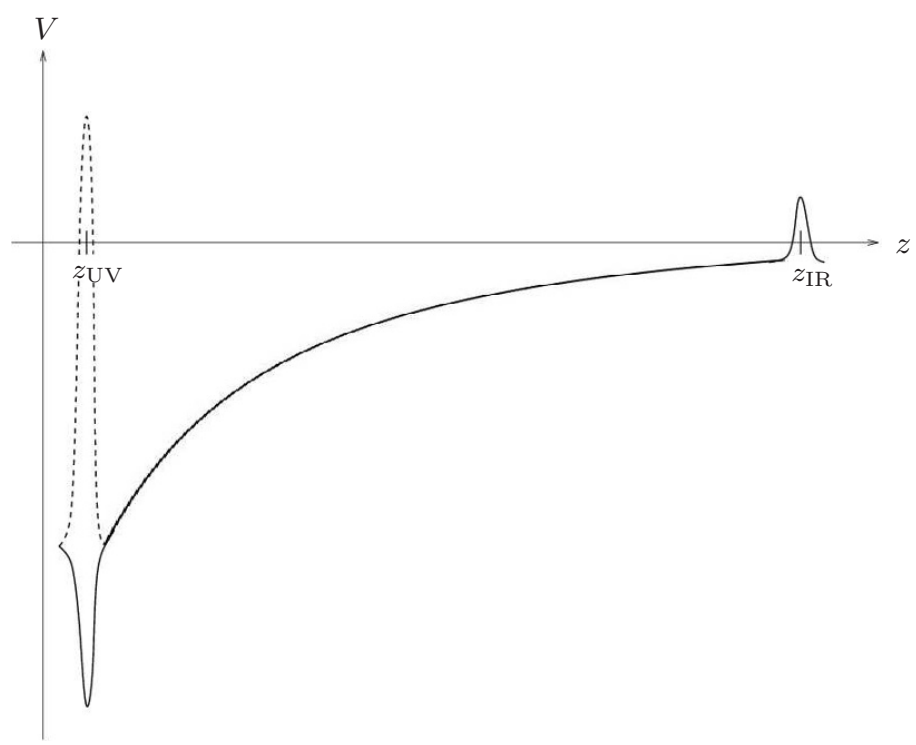

Figure 2. Potential in the effective Schrödinger equation for a $5 \mathrm{~d}$ mass $M^{2}=-4 k^{2}$. The dotted $\delta$-peak appears when a large mass term on the UV brane is added.

such that, for $\lambda>\frac{3}{2}$, the $\delta$-peak at the UV brane becomes repulsive (cf. figure 2). It is intuitively clear that such a modification of the potential lifts the energy eigenvalues and can therefore remove tachyonic modes.

Indeed, it will become apparent in the next section that, in the limit $z_{\mathrm{IR}} \rightarrow \infty$ (the RS II limit [44]), a zero mode appears if $\lambda=\lambda_{0} \equiv 2-\alpha$. This is the minimal value of $\lambda$ required for stability - for $\lambda>\lambda_{0}$ all modes are massive. In the RS I case (for finite $z_{\mathrm{IR}}$ ) the minimal value of $\lambda$ may differ from $\lambda_{0}$, depending on the specific boundary condition imposed at the IR brane. However, this difference goes to zero together with $z_{\mathrm{UV}} / z_{\mathrm{IR}}$ and its precise value will not be important. Moreover, for the specific case of supersymmetric boundary conditions (when the IR brane carries as mass term of opposite value w.r.t. the UV-brane mass term [31]), the minimal value continues to be $\lambda_{0}$. Thus, it will be convenient for us to 
assume such supersymmetric IR boundary conditions (modifying eq. (2.10) appropriately) and to think of $\lambda_{0}=2-\alpha$ as of the minimal value of $\lambda$, also at finite $z_{\mathrm{IR}}$.

To conclude our preliminary discussion of tachyons in a slice of $\mathrm{AdS}_{5}$, we briefly describe the KK mass spectrum. As long as $\lambda>\lambda_{0}$, the spectrum does not differ qualitatively from the familiar massless or positive-mass-squared case. As we already emphasized, we are dealing with a quantum-mechanical problem on a compact space of size $\sim\left(z_{\mathrm{IR}}-z_{\mathrm{UV}}\right) \simeq z_{\mathrm{IR}}$. We thus naively ${ }^{5}$ expect $m_{n}$ to be quantized in units of $z_{\mathrm{IR}}^{-1}$, to which we will from now on refer as our IR scale $m_{\mathrm{IR}} \equiv z_{\mathrm{IR}}^{-1}=k e^{-k \ell}$. Indeed, the Bessel function analysis of appendix A shows that $m_{n} \simeq\left(n+\frac{\alpha}{2}-\frac{3}{4}\right) \pi k e^{-k \ell}$ for $1 \ll n \ll e^{k \ell}$. This approximate formula remains rather accurate all the way down to relatively small $n$.

We finally note that a technically related analysis of the wave functions of a tachyonic scalar has recently appeared in [39] in a rather different physical context. We refer to that paper for more details on the quantum mechanical analogue and corresponding references.

\section{Decays mediated by $\mathrm{AdS}_{5}$ fields}

As one can see from figure 1, a massless scalar has to tunnel through an effective potential barrier before it can reach the UV brane. ${ }^{6}$ By contrast, a tachyonic scalar with $M^{2}=-4 k^{2}$ has no such barrier to surmount (cf. figure 2). Thus, one expects the tachyon to couple more strongly to the UV brane. There is, however, a compensating effect: The UV-brane mass term of the tachyon suppresses its value at the brane. To determine the relative importance of these two effects, we now estimate the decay rate of the KK modes of a bulk scalar to a gauge theory living on the UV brane. Our interest is in the dependence on the parameters $\alpha$ and $\lambda$.

\subsection{Generic UV-brane mass term}

We assume that $\Phi$ interacts with a gauge theory localized at the UV brane via the action ${ }^{7}$

$$
\int d^{4} x d z \sqrt{-G} k^{-\frac{3}{2}} \Phi(x, z) \operatorname{tr}\left(F^{\mu \nu} F_{\mu \nu}\right) \delta\left(z-z_{\mathrm{UV}}\right) .
$$

We have arbitrarily set the coefficient of this interaction term to one in units of $k$ since we will anyway perform only an order-of-magnitude calculation. Using eq. (2.4), we see that the coupling of the $n$-th KK mode to the gauge theory is given by

$$
\int d^{4} x k^{-\frac{3}{2}} \varphi_{n}\left(z_{\mathrm{UV}}\right) \operatorname{tr}\left(F^{\mu \nu} F_{\mu \nu}\right) \chi_{n}(x) .
$$

\footnotetext{
${ }^{5}$ This is naive in the sense that the potential introduces the further dimensionful quantity $z_{\mathrm{UV}}$ into the calculation. However, in the limit $z_{\mathrm{UV}} / z_{\mathrm{IR}} \rightarrow 0$ the $1 / z^{2}$ term together with the UV-brane $\delta$ function basically just serve to provide a certain boundary condition at one side of the interval. Hence the scale $z_{\mathrm{UV}}$ does not affect the (low-lying part of) the spectrum. This will become apparent after the analysis of the next section.

${ }^{6}$ This is the same situation as for the graviton (cf. [44]).

${ }^{7}$ As we will discuss in section 6 in more detail, this term can be viewed as an effective $5 \mathrm{~d}$ description of the dilaton coupling to the gauge fields on a D-brane stack.
} 
The coupling constant between the $n$-th KK mode and two gauge bosons,

$$
g_{n} \sim k^{-\frac{3}{2}} \varphi_{n}\left(z_{\mathrm{UV}}\right)=k^{-\frac{3}{2}} \psi_{n}\left(z_{\mathrm{UV}}\right),
$$

has mass dimension $\left[g_{n}\right]=-1$ and determines the decay rate according to

$$
\Gamma_{n} \sim g_{n}^{2} m_{n}^{3} .
$$

In order to calculate $g_{n}$, we need a detailed understanding of the shape of the KK modes. To achieve this, we introduce the coordinate $\widehat{z}=m_{n} z$ (for each $m_{n} \neq 0$ ) and translate the $\delta$ functions back to boundary conditions at $\widehat{z}_{\mathrm{UV}}$ and $\widehat{z}_{\mathrm{IR}}$. We then have to solve

$$
\left[\partial_{\widehat{z}}^{2}+1-\left(\frac{\alpha^{2}-\frac{1}{4}}{\widehat{z}^{2}}\right)\right] \psi_{n}(\widehat{z})=0,
$$

subject to

$$
\left.\left(\widehat{z} \partial_{\widehat{z}} \psi_{n}\right)\right|_{\widehat{z}=\widehat{z}_{U V}}=\left.\left(\lambda-\frac{3}{2}\right) \psi_{n}\right|_{\widehat{z}=\widehat{z}_{U V}} \quad \text { and }\left.\quad\left(\widehat{z} \partial_{\widehat{z}} \psi_{n}\right)\right|_{\widehat{z}=\widehat{z}_{I R}}=\left.\left(\lambda-\frac{3}{2}\right) \psi_{n}\right|_{\widehat{z}=\widehat{z}_{I R}}
$$

where we have also modified the IR boundary condition as mentioned earlier.

It is straightforward to solve eq. (3.5) separately in the regions $\widehat{z} \ll 1$ and $\widehat{z} \gg 1$, where the first and second term of the potential, respectively, can be neglected. ${ }^{8}$ We find that for small $\widehat{z}$

$$
\psi_{n, \mathrm{UV}}(\widehat{z}) \simeq \begin{cases}\frac{1}{N_{\alpha}}\left(\widehat{z}^{\frac{1}{2}+\alpha}+B_{\alpha} \widehat{z}^{\frac{1}{2}-\alpha}\right) & \text { for } \alpha>0 \\ \frac{1}{N_{0}}\left(\widehat{z}^{\frac{1}{2}}+B_{0} \widehat{z}^{\frac{1}{2}} \ln \frac{1}{\widehat{z}}\right) & \text { for } \alpha=0,\end{cases}
$$

while for large $\widehat{z}$

$$
\psi_{n, \mathrm{IR}}(\widehat{z}) \simeq \frac{1}{A_{\alpha}} \cos \left(\widehat{z}+C_{\alpha}\right),
$$

where $N_{\alpha}, B_{\alpha}, A_{\alpha}$ and $C_{\alpha}$ are constants of integration. ${ }^{9}$ The qualitative behaviour of the resulting wave function is sketched in figure 3 .

The canonical normalization of $\chi_{n}$ implies, together with eq. (2.6), the normalization condition

$$
\frac{1}{m_{n}} \int_{\widehat{z}_{\mathrm{UV}}}^{\widehat{z}_{\mathrm{IR}}} d \widehat{z} \psi_{n}(\widehat{z})^{2}=1
$$

for our wave function. Let us assume that $\widehat{z}_{\mathrm{IR}} \gg 1$ and that the absolute value of the wave function grows between $\widehat{z}_{\mathrm{UV}}$ and $\widehat{z} \sim 1$ (this turns out to always be the case if $\lambda$ is generic).

\footnotetext{
${ }^{8}$ We assume $\alpha^{2}-\frac{1}{4} \sim \mathcal{O}(1)$ for simplicity, excluding the special region $\alpha \simeq \frac{1}{2}$.

${ }^{9}$ Note that, with the redefinitions

$$
N_{0} \equiv \frac{N_{\alpha}}{1+B_{\alpha}} \quad \text { and } \quad B_{0} \equiv \frac{\alpha\left(1-B_{\alpha}\right)}{1+B_{\alpha}},
$$

the second line of eq. (3.7) can be recovered as the $\alpha \rightarrow 0$ limit of the first line.
} 


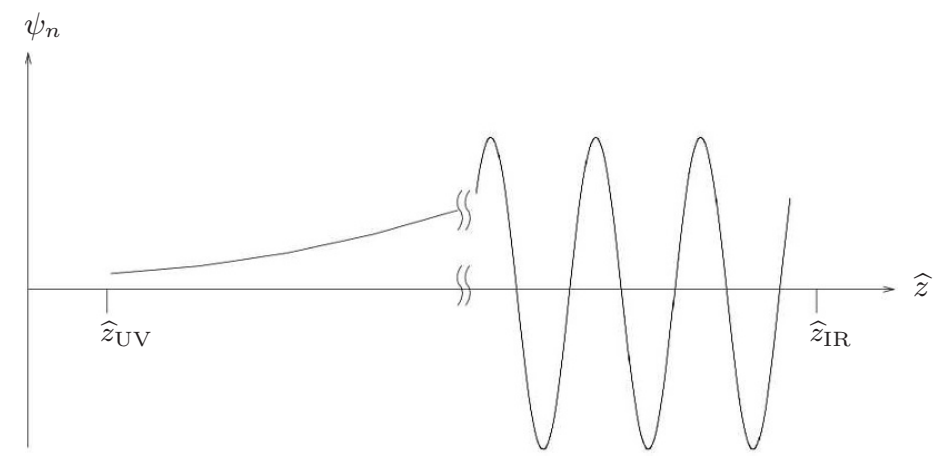

Figure 3. Sketch of the wavefunction $\psi_{n}$ as a function of $\widehat{z}$

It is then apparent that the overall normalization is dominated by the cosine solution in the $\mathrm{IR}$ region $1 \leq \widehat{z} \leq \widehat{z}_{\mathrm{IR}}$, implying

$$
A_{\alpha} \sim \sqrt{\widehat{z}_{\mathrm{IR}} / m_{n}}=m_{\mathrm{IR}}^{-1 / 2} .
$$

Of course, we are actually interested in the lowest-lying modes, so that $z_{\mathrm{IR}} \sim \mathcal{O}(1)$ rather than $z_{\mathrm{IR}} \gg 1$. Nevertheless, the approximate cosine region (which in this case contains only a few oscillations) continues to contribute an $\mathcal{O}(1)$ fraction to the total normalization. Thus, eq. (3.10) continues to be correct up to $\mathcal{O}(1)$ factors.

To summarize, we now know that the UV solution has to be matched to the IR solution with approximate value $A_{\alpha}^{-1} \sim m_{\mathrm{IR}}^{1 / 2}$ at $\widehat{z} \sim 1$. For the decay rate, we need the UV-brane value of this UV solution, which we will now derive.

The first boundary condition of eq. (3.6) together with eq. (3.7) gives

$$
\begin{aligned}
& B_{\alpha}=-\left(1-\frac{2 \alpha}{\lambda-2+\alpha}\right) \widehat{z}_{\mathrm{UV}}^{2 \alpha}, \\
& B_{0}=-\left(1-\frac{1}{(\lambda-2) \ln \left(1 / \widehat{z}_{\mathrm{UV}}\right)+1}\right) \frac{1}{\ln \left(1 / \widehat{z}_{\mathrm{UV}}\right)} .
\end{aligned}
$$

Now, for $\lambda=\lambda_{0}+\mathcal{O}(1)$ and $\alpha=\mathcal{O}(1)$, we see that both the $\widehat{z}^{\frac{1}{2}+\alpha}$ solution and the $\widehat{z}^{\frac{1}{2}-\alpha}$ solution, as well as the full solution $\psi_{n, \mathrm{UV}}$ are of comparable size at $\widehat{z}_{\mathrm{UV}}$. Thus, only the more strongly growing solution is important for the matching at $\widehat{z} \sim 1$. The value of $\psi_{n}$ at the UV brane is suppressed according to the behaviour of this solution. By contrast, for $\alpha=0$ the $\widehat{z}^{\frac{1}{2}}$ solution and the $\widehat{z}^{\frac{1}{2}} \ln (1 / \widehat{z})$ solution cancel almost exactly at the UV brane, leading to an extra suppression factor $1 / \ln (1 / \widehat{z})$ in the brane value of $\psi_{n}$. In summary, we have

$$
\psi_{n}\left(\widehat{z}_{\mathrm{UV}}\right) \sim \begin{cases}\widehat{z}_{\mathrm{UV}}^{\frac{1}{2}+\alpha} m_{\mathrm{IR}}^{\frac{1}{2}} & \text { for } \alpha>0 \\ \frac{\widehat{z}_{\mathrm{UV}}^{\frac{1}{2}}}{\ln \left(1 / \widehat{z}_{\mathrm{UV}}\right)} m_{\mathrm{IR}}^{\frac{1}{2}} & \text { for } \alpha=0 .\end{cases}
$$

Note that the $\alpha=0$ result could also have been derived by using the expression for $B_{\alpha}$ in eq. (3.11) and carefully taking the limit $\alpha \rightarrow 0$. It is valid for $\alpha \lesssim \ln \left(1 / \widehat{z}_{\mathrm{UV}}\right)^{-1}$. 
Using eqs. (3.3) and (3.4) and recalling that $\widehat{z}_{\mathrm{UV}}=m_{n} / k$, one finds the decay rates

$$
\Gamma_{n} \sim \begin{cases}\left(\frac{m_{n}}{k}\right)^{4+2 \alpha} m_{\mathrm{IR}} & \text { for } \alpha>0 \\ \left(\frac{m_{n}}{k}\right)^{4} \frac{m_{\mathrm{IR}}}{\ln ^{2}\left(k / m_{n}\right)} & \text { for } \alpha=0\end{cases}
$$

These rates represent one of our main results and will be used in the cosmological applications later on.

\subsection{Tuned UV-brane mass term}

Equation (3.11) suggests that the point $\lambda=\lambda_{0}=2-\alpha$ may require special attention: Near this point, the UV-brane value of the $\widehat{z}^{\frac{1}{2}-\alpha}$ mode (the falling or weakly growing mode) is significantly enhanced compared to the value of the $\widehat{z}^{\frac{1}{2}+\alpha}$ mode (the strongly growing mode). We already know that, for $\lambda=\lambda_{0}$, the lowest mode is massless since, for the specific choice of IR boundary conditions discussed earlier, $\lambda_{0}$ is the minimal allowed value. This mode can not decay unless the tuning is imperfect. We will not discuss the possible decay rate of such an 'almost massless' mode.

Furthermore, in the tuned case the UV-brane $\delta$-function is least-repulsive (or most attractive). Thus, we expect the decay rates of higher modes to be larger for $\lambda=\lambda_{0}$ than for the generic case of $\lambda>\lambda_{0}$. These enhanced decay rates are our main interest in this subsection.

Let us first consider the case $0<\alpha<1$ and $\lambda=\lambda_{0}$. Taking eq. (3.11) at face value, $B_{\alpha}$ is infinite, which simply means that in the solution of eq. (3.7) only the $\widehat{z}^{\frac{1}{2}-\alpha}$ term is present. When moving from $\widehat{z}=\mathcal{O}(1)$ to $\widehat{z}=\widehat{z}_{\mathrm{UV}}$, the wave function then changes by a factor $\widehat{z}_{\mathrm{UV}}^{\frac{1}{2}-\alpha}$. Repeating the arguments which lead us to eq. (3.14), this gives the decay rate

$$
\Gamma_{n} \sim\left(\frac{m_{n}}{k}\right)^{4-2 \alpha} m_{\mathrm{IR}} \text { for } 0<\alpha<1 \text { and } \lambda=\lambda_{0} .
$$

In the case $\alpha=0$, we have $B_{0}=0$ at $\lambda=\lambda_{0}$ according to eq. (3.12). Hence the wave function falls by a factor $\widehat{z}_{\mathrm{UV}}^{\frac{1}{2}}$. This implies

$$
\Gamma_{n} \sim\left(\frac{m_{n}}{k}\right)^{4} m_{\mathrm{IR}} \quad \text { for } \quad \alpha=0 \text { and } \quad \lambda=\lambda_{0} .
$$

As expected, a tuned value of $\lambda$ enhances the rates in the whole range $0 \leq \alpha<1$, with the enhancement being more pronounced at larger $\alpha$.

Apparently, nothing in the above calculation depends on the restriction to $\alpha<1$. However, it is easy to see that something goes wrong for $\alpha>1$. Indeed, if only the $\widehat{z}^{\frac{1}{2}-\alpha}$ mode is relevant for all $\widehat{z} \ll 1$, the $\psi_{n}^{2}$ normalization integral is dominated by the region near $\widehat{z}_{\mathrm{UV}}$. Thus, the relevant mode is UV localized. Obviously, this can not be the case for all modes with $m_{n} \ll k$ (to which our argument formally applies).

At the technical level, the above problem comes from the insufficient accuracy of the approximation made in eq. (3.7). This accuracy can be improved by taking into account 
higher-order terms in the small-argument expansions of the Bessel functions. ${ }^{10}$ Including only the first-order correction, we find

$$
\psi_{n, \mathrm{UV}}(\widehat{z}) \simeq \begin{cases}\frac{1}{N_{\alpha}}\left[\widehat{z}^{\frac{1}{2}+\alpha}\left(1-\frac{\widehat{z}^{2}}{4(1+\alpha)}\right)+B_{\alpha} \widehat{z}^{\frac{1}{2}-\alpha}\left(1-\frac{\widehat{z}^{2}}{4(1-\alpha)}\right)\right] & \text { for } \alpha \neq 1 \\ \frac{1}{N_{1}}\left[\widehat{z}^{\frac{3}{2}}\left(1-\frac{\widehat{z}^{2}}{8}\right)+B_{1} \widehat{z}^{-\frac{1}{2}}\left(1+\frac{\widehat{z}^{2} \ln (1 / \widehat{z})}{2}\right)\right] & \text { for } \alpha=1 .\end{cases}
$$

In fact, the $\widehat{z}^{2}$ correction to the $\widehat{z}^{\frac{1}{2}+\alpha}$ solution will not play any role and we drop it in the following. With this simplification, eq. (3.17) together with the UV boundary conditions implies

$$
\begin{aligned}
B_{\alpha} & =4 \alpha(1-\alpha) \widehat{z}_{\mathrm{UV}}^{2 \alpha-2} \\
B_{1} & =\frac{4}{2 \ln \left(1 / \widehat{z}_{\mathrm{UV}}\right)+1}
\end{aligned}
$$

This result can be intuitively understood as follows: It is clear that, for generic $\lambda$, the boundary condition eq. (3.6) can only be fulfilled if both solutions to the wave equation, $\widehat{z}^{\frac{1}{2}+\alpha}+\cdots$ and $B_{\alpha} \widehat{z}^{\frac{1}{2}-\alpha}+\cdots$, are of comparable size at the UV brane. This immediately gives $B_{\alpha} \sim \widehat{z}_{\mathrm{UV}}^{2 \alpha}$ as in eq. (3.11). Now, for tuned $\lambda_{0}=2-\alpha$, the function $B_{\alpha} \widehat{z}^{\frac{1}{2}-\alpha}$ fulfills the boundary condition already by itself, as can be easily checked. Therefore, the boundary condition now forces the leading correction to that function, $B_{\alpha} \widehat{z}^{\frac{5}{2}-\alpha}$, to have the same size as the other solution, $\widehat{z}^{\frac{1}{2}+\alpha}$, at the UV brane. This gives $B_{\alpha} \sim \widehat{z}_{\mathrm{UV}}^{2 \alpha-2}$ in agreement with eq. (3.18).

We now observe that, as long as $\alpha<1$, the $\widehat{z}^{\frac{1}{2}-\alpha}$ solution is dominant everywhere between $\widehat{z}=\widehat{z}_{\mathrm{UV}}$ and $\widehat{z}=\mathcal{O}(1)$. This justifies our previously derived rate of eq. (3.15) and shows that the presently discussed $\widehat{z}^{2}$ corrections are not important for $0 \leq \alpha<1$.

Next, we focus on the regime $\alpha>1$. In this case, we see that the $\widehat{z}^{\frac{1}{2}-\alpha}$ solution dominates for $\widehat{z}_{\mathrm{UV}}<\widehat{z}<\widehat{z}_{\mathrm{UV}}^{1-1 / \alpha}$. By contrast, the $\widehat{z}^{\frac{1}{2}+\alpha}$ solution is larger for $\widehat{z}_{\mathrm{UV}}^{1-1 / \alpha}<$ $\widehat{z}<\mathcal{O}(1)$. Although figure 3 does not describe this regime correctly, one can check that the normalization is nevertheless dominated by the IR region. It is then easy to see that

$$
\psi_{n}\left(\widehat{z}_{\mathrm{UV}}\right) \sim z_{\mathrm{UV}}^{\alpha-\frac{3}{2}} m_{\mathrm{IR}}^{\frac{1}{2}} \quad \text { for } \quad \alpha>1 \text { and } \lambda=\lambda_{0},
$$

giving the decay rate

$$
\Gamma_{n} \sim\left(\frac{m_{n}}{k}\right)^{2 \alpha} m_{\mathrm{IR}} \quad \text { for } \quad \alpha>1 \text { and } \quad \lambda=\lambda_{0} .
$$

It can be checked that, in spite of the extra logarithms appearing at intermediate steps, the special case $\alpha=1$ is correctly reproduced by simply taking the appropriate limit of either eq. (3.15) or eq. (3.21).

The $5 \mathrm{~d}$ graviton with indices $\mu, \nu$ has the same equation of motion as a massless $5 \mathrm{~d}$ scalar without boundary mass (i.e. for $\lambda=\lambda_{0}=0$ ). We assume $M_{5} \sim k$ in the following.

\footnotetext{
${ }^{10}$ Alternatively, they can be determined by solving eq. (3.5) iteratively, treating the ' 1 ' as a perturbation.
} 
The coupling of the $5 \mathrm{~d}$ graviton to the energy-momentum tensor on the UV brane is then suppressed by a factor $k^{-3 / 2}$ as in eq. (3.1). Hence eq. (3.21) with $\alpha=2$ also applies to the graviton and we have

$$
\Gamma_{n} \sim\left(\frac{m_{n}}{k}\right)^{4} m_{\mathrm{IR}} \quad \text { for graviton KK modes, }
$$

in agreement with the literature on graviton tunneling between two throats (see e.g. [12, 45, 46]). These results are relevant since the throat to which the KK modes decay is dual to a UV-brane-localized gauge theory [46]. Of course, eq. (3.22) corresponds to the case where this gauge theory has $\mathcal{O}(1)$ degrees of freedom. We finally note that the coupling strength of graviton KK modes to the UV brane has been given, e.g., in appendix A of [103]. For $M_{5} \sim k$, it reads $g_{n} \sim \sqrt{m_{n} m_{\mathrm{IR}}} / k^{2}$. The decay rate then follows from eq. (3.4) and also reproduces eq. (3.22).

\section{Situations dominated by the tachyon}

A detailed discussion of the application of our 5d analysis to the Klebanov-Strassler throat is the subject of section 6 . However, to explain the relevance of tachyon decay rates we have to jump somewhat ahead and mention certain facts concerning warped flux compactifications already in this section: In flux compactifications, scalars obtain a large mass in the unwarped part of the compact space. ${ }^{11}$ In our $5 \mathrm{~d}$ model, this corresponds to a large and generically detuned mass term on the UV brane. It is then evident from eq. (3.14) that a maximally tachyonic scalar $(\alpha=0)$ has a considerably larger decay rate than a massless scalar $(\alpha=2)$. This can be easily understood from the quantum mechanical analog: The suppression effect of the UV mass term is present for both fields, but only the massless scalar has to surmount an additional potential barrier. Thus, assuming that a certain KK mode decays only via scalars and that all relevant scalars have a large UV-brane mass term, the tachyon governs the decay. Clearly, the assumption that decays proceed only through scalars is non-trivial: The graviton decay rate, eq. (3.22) is larger than the tachyon rate, eq. (3.14) (albeit only by a factor $\left.\left(\ln \left(k / m_{n}\right)\right)^{-2} \sim(k \ell)^{-2}\right)$. However, this is only relevant for spin-two KK modes which can mix with the graviton.

Next, we observe that there are specific situations where, in contrast to what was said above, the tachyon decay rate is larger than that of the graviton. This is possible because the tachyon wavefunction rises while the graviton wavefunction falls when moving away from the UV brane. Thus, if there is a probe brane in the throat which is localized somewhere between the UV and IR brane, the decay to this probe brane can be dominated by the tachyon (even if a corresponding graviton decay is allowed). We use the simple ansatz eq. (3.1) for the coupling to gauge fields on the probe brane at $\widehat{z}_{\delta}$ (where $\widehat{z}_{\mathrm{UV}}<\widehat{z}_{\delta}<\widehat{z}_{\mathrm{IR}}$ ). ${ }^{12}$

\footnotetext{
${ }^{11}$ We assume that the Kähler moduli are stabilized as well (which requires effects other than flux).

${ }^{12} \mathrm{As}$ a string-theoretic realization of this situation, consider a D7 brane wrapping a 3-cycle of the $T^{1,1}$ in a Klebanov-Strassler throat and extending from the compact Calabi-Yau to a certain lowest point in the throat. For a discussion of such embeddings and applications see e.g. [47-50]. Even though, in 5d language, the D7-brane gauge theory lives everywhere between $\widehat{z}_{\mathrm{UV}}$ and $\widehat{z}_{\delta}$, we model this situation by a gauge theory localized at $\widehat{z}_{\delta}$. This is a reasonable approximation since the coupling to throat fields is dominated by interactions at the largest relevant values of $\widehat{z}$.
} 
We now demonstrate the enhancement of the decay rates quantitatively. Let the probe brane be localized at $y=\delta$ for some $\delta \ll \ell$. For the light modes, $\widehat{z}_{\delta}=\widehat{z}_{\mathrm{UV}} e^{k \delta} \sim n e^{-k(\ell-\delta)}$ is small and we can use the approximate wavefunctions of eq. (3.7). We see that, as $\widehat{z}_{\delta}$ increases, the tachyon wave function $(\alpha=0)$ grows like $\widehat{z}^{\frac{1}{2}}$ (we neglect the additional logarithmic dependence). By contrast, the KK graviton wave function $(\alpha=2)$ falls like $\widehat{z}^{\frac{1}{2}-\alpha}$. This is because, as explained before eq. (3.20), the falling solution dominates near the UV brane. Thus, the ratio of the two wave functions at $\widehat{z}_{\delta}$ is enhanced, relative to its UV brane value, by a factor $\left(\widehat{z}_{\delta} / \widehat{z}_{\mathrm{UV}}\right)^{2}$. Given that the tachyon decay rate to the UV brane is suppressed relative to the corresponding graviton rate by the logarithmic factor mentioned above, we conclude that

$$
\frac{\Gamma_{\text {tachyon }}}{\Gamma_{\text {graviton }}} \sim \frac{\left(\widehat{z}_{\delta} / \widehat{z}_{\mathrm{UV}}\right)^{4}}{\ln ^{2}\left(\widehat{z}_{\mathrm{IR}} / \widehat{z}_{\mathrm{UV}}\right)}
$$

for decays to the probe brane. Thus, the tachyon starts to dominate the decay when the probe-brane warp factor $\left(\widehat{z}_{\delta} / \widehat{z}_{\mathrm{UV}}\right)^{-1}$ becomes smaller than $1 / \sqrt{\ln \left(\widehat{z}_{\mathrm{IR}} / \widehat{z}_{\mathrm{UV}}\right)}$. This is obviously a very weak requirement.

Finally, we mention a third situation in which the tachyon dominates the decay rates to the UV sector. We can not argue from the $5 \mathrm{~d}$ perspective how natural or unnatural it is to have a tuned UV mass term, $\lambda=\lambda_{0}$. (We will return to this question in the string-theoretic context in section 6). However, a tuned value of $\lambda_{0}$ is clearly a legitimate possibility. Combining eqs. (3.15) and (3.21), the corresponding decay rates can be written as

$$
\Gamma_{n} \sim\left(\frac{m_{n}}{k}\right)^{2+2|\alpha-1|} m_{\mathrm{IR}} \quad \text { for } \quad \lambda=\lambda_{0} .
$$

For $0<\alpha<2$, this obviously dominates the decay rates corresponding to massless scalars and the graviton. The maximal enhancement is realized for $\alpha=1$, i.e., for a tachyon the negative mass-squared of which is half that of the Breitenlohner-Freedman bound.

\section{CFT interpretation}

We now present physical arguments for the decay rates of section 3 using the 'CFT-dual' description of a Randall-Sundrum model as a strongly coupled 4d field theory. In other words, we are going to appeal to a simplified, purely field-theoretic version of the AdS/CFT correspondence [51]. In this description, a scalar bulk field with mass squared $M^{2}$ corresponds to a CFT operator $\mathcal{O}_{\Delta}$ with conformal dimension $\Delta=2+\sqrt{4+M^{2} / k^{2}}=2+\alpha .{ }^{13}$ Without a UV brane, the generating functional of CFT correlators can be obtained from the gravity description via the relation $[54,55]$

$$
\int \mathcal{D} \phi_{\mathrm{CFT}} e^{-S_{\mathrm{CFT}}\left[\phi_{\mathrm{CFT}}\right]-\int d^{4} x \phi_{0} \mathcal{O}_{\Delta}}=\int_{\phi_{0}} \mathcal{D} \Phi e^{-S_{5 d}[\Phi]} .
$$

Here $\phi_{0}(x)$ specifies the behaviour of the bulk field $\Phi(x, z)$ near the boundary of $\mathrm{AdS}_{5}$ (taking $z \rightarrow 0$ ) to be $\Phi(x, z) \simeq \phi_{0}(x) z^{4-\Delta} k^{3 / 2}$. It thus acts as a source for the correspond-

\footnotetext{
${ }^{13}$ As we will discuss below, for $\alpha<1$, also the relation $\Delta=2-\alpha$ can be realized [53].
} 
ing operator in the generating functional. ${ }^{14}$ The value of $\phi_{0}$ is kept fixed in the functional integral on the right-hand side and $\phi_{\mathrm{CFT}}$ denotes the dynamical CFT fields.

In the Randall-Sundrum model, there is a brane at $z=k^{-1}$, corresponding to a UV cutoff $\Lambda_{\mathrm{UV}}=k$ in the CFT. The source $\phi_{0}(x)=k^{5 / 2-\Delta} \Phi\left(x, k^{-1}\right)$ now becomes a physical degree of freedom and has to be included in the functional integral [57]:

$$
\int \mathcal{D} \phi_{0} e^{-S_{\mathrm{UV}}\left[\phi_{0}\right]} \int_{\Lambda_{\mathrm{UV}}} \mathcal{D} \phi_{\mathrm{CFT}} e^{-S_{\mathrm{CFT}}\left[\phi_{\mathrm{CFT}}\right]-\int d^{4} x \phi_{0} \mathcal{O}_{\Delta}}=\int \mathcal{D} \phi_{0} e^{-S_{\mathrm{UV}}\left[\phi_{0}\right]} \int_{\phi_{0}} \mathcal{D} \Phi e^{-S_{5 d}[\Phi]}
$$

We may think of the 1.h. side of eq. (5.2) as being defined by the r.h. side. In our case, the UV-brane action of the bulk scalar field, $S_{\mathrm{UV}}\left[\phi_{0}\right]$, consists of the UV-brane mass term and the interaction term with the brane-localized gauge theory. (We have suppressed the gauge fields in the functional integral in eq. (5.2) for notational simplicity.) Thus, at the UV scale $\Lambda_{\mathrm{UV}}$, the complete Lagrangian on the CFT side of the duality reads

$$
\mathcal{L}=\mathcal{L}_{\mathrm{CFT}}\left[\phi_{\mathrm{CFT}}\right]+M_{\phi_{0}}^{2} \phi_{0}^{2}+\frac{1}{\Lambda_{\mathrm{UV}}^{\Delta-3}} \phi_{0} \mathcal{O}_{\Delta}+\frac{1}{\Lambda_{\mathrm{UV}}} \phi_{0} \operatorname{tr}\left(F^{\mu \nu} F_{\mu \nu}\right)
$$

where we have redefined $\phi_{0}$ by an appropriate power of $\Lambda_{\mathrm{UV}}$ to give it mass dimension one.

When running the action corresponding to eq. (5.3) down to scales below $\Lambda_{\mathrm{UV}}$, the CFT induces a kinetic term $\sim\left(\partial \phi_{0}\right)^{2}$ for the scalar $\phi_{0}$, which now manifestly becomes a propagating field [56]. Similarly, the tachyonic instability which arises for $M^{2}<0$ in the presence of a UV brane can be understood as an effect of CFT-induced corrections. In this language, loop effects of the CFT drive a scalar $\phi_{0}$ with $M_{\phi_{0}}^{2}=0$ at the UV scale to a negative mass squared at the IR scale if $\Delta<4$ [38]. A sufficiently large mass term at the UV scale prevents the CFT from making the scalar tachyonic. In particular, the tuning $\lambda=\lambda_{0}$ of the UV mass described earlier can be interpreted in the CFT language as a tuning of $M_{\phi_{0}}^{2}$ which ensures that $\phi_{0}$ becomes massless at the IR scale.

We first focus on the case of a generic, large UV mass term, $\lambda=\lambda_{0}+\mathcal{O}(1)$. In this case, $\phi_{0}$ has a mass of the order $\Lambda_{\mathrm{UV}}$ even after running down to the IR scale $m_{\mathrm{IR}}$. Since $\phi_{0}$ remains heavy all the way from $\Lambda_{\mathrm{UV}}$ down to $m_{\mathrm{IR}}$, its effect on the CFT dynamics and, in particular, on the dimension of $\mathcal{O}_{\Delta}$ is negligible. Thus, the IR scale Lagrangian is still that of eq. (5.3), modified only by a kinetic term and a mass correction for $\phi_{0}$. We can now integrate out $\phi_{0}$ entirely, finding the coupling

$$
\sim \frac{1}{\Lambda_{\mathrm{UV}}^{\Delta-2}} \frac{1}{M_{\phi_{0}}^{2}} \mathcal{O}_{\Delta} \operatorname{tr}\left(F^{\mu \nu} F_{\mu \nu}\right)
$$

between the brane-localized gauge theory and the operator $\mathcal{O}_{\Delta}$. At low scales, the conformal symmetry of $\mathcal{L}_{\mathrm{CFT}}$ is broken by the IR brane. In the case of the Klebanov-Strassler throat, this breaking of (approximate) conformal invariance is a dynamical effect within the strongly-coupled gauge theory. The low-lying glueball states of this gauge theory correspond to (linear combinations $[58,59]$ of) the KK modes of the bulk fields. Assuming

\footnotetext{
${ }^{14}$ More precisely, $\Phi(x, z) \simeq \phi_{0}(x) z^{4-\Delta} k^{3 / 2}+A(x) z^{\Delta} k^{3 / 2}$, where $A(x)$ can be interpreted as the expectation value of the dual operator $\mathcal{O}_{\Delta}$ and $\phi_{0}(x)$ as the source. The factors $k^{3 / 2}$ have been introduced for dimensional reasons.
} 


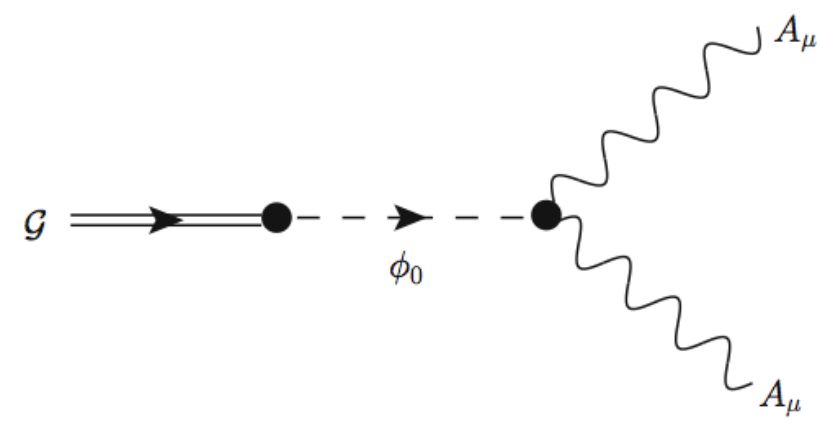

Figure 4. Decay of a glueball $\mathcal{G}$ via the light field $\phi_{0}$ into two gauge fields $A_{\mu}$.

that these glueballs have non-vanishing overlap with $\mathcal{O}_{\Delta}$, they decay to the brane-localized gauge theory via the operator in eq. (5.4). Since we assumed that $M_{\phi_{0}}^{2} \sim \Lambda_{\mathrm{UV}}^{2}$ even at the IR scale, the decay rate

$$
\Gamma \sim\left(\frac{m_{\mathrm{IR}}}{\Lambda_{\mathrm{UV}}}\right)^{2 \Delta} m_{\mathrm{IR}}
$$

follows by dimensional analysis. Recalling that $\Lambda_{\mathrm{UV}}=k$ and $\Delta=2+\alpha$, we see immediately that this agrees with the first line of eq. (3.14).

We now turn our attention to the tuned case, $\lambda=\lambda_{0}$, making use of the holographic picture developed in $[38,57]$. As before, we run the Lagrangian of eq. (5.3) down to the scale $m_{\mathrm{IR}}$. In contrast to our previous discussion, we can not appeal to the large mass of $\phi_{0}$ to ignore its influence on the CFT. Indeed, as explained earlier, $M_{\phi_{0}}^{2}$ approaches zero as the energy scale approaches $m_{\mathrm{IR}}$. However, provided that $\alpha>1(\Delta>3)$, we can now argue that the influence of $\phi_{0}$ is negligible because of its small mixing with CFT states. Indeed, for $\Delta>3$, the operator $\phi_{0} \mathcal{O}_{\Delta}$ is irrelevant. Thus, we assume that the IR scale effective action contains light glueballs and a light field $\phi_{0}$, with a mixing term suppressed by $1 / \Lambda_{\mathrm{UV}}^{\Delta-3}$.

The decay of glueballs to fields of the UV-brane gauge theory now proceeds via the massless or light 'source field' $\phi_{0}$, cf. figure 4 . The rate follows by dimensional analysis:

$$
\Gamma \sim\left(\frac{m_{\mathrm{IR}}}{\Lambda_{\mathrm{UV}}}\right)^{2 \Delta-4} m_{\mathrm{IR}}
$$

This result can also be immediately obtained from eq. (5.5): That equation secretly contains a suppression factor $1 /\left(\Lambda_{\mathrm{UV}}^{2}\right)^{2}$ coming from the massive $\phi_{0}$ propagator. Replacing this mass by $m_{\mathrm{IR}}$, i.e. multiplying eq. (5.5) by $\left(\Lambda_{\mathrm{UV}} / m_{\mathrm{IR}}\right)^{4}$, we obtain eq. (5.6). This rate agrees with the result of the gravity calculation for $\alpha>1$ given in eq. (3.21).

For $\alpha<1(\Delta<3)$ the above line of argument breaks down since the mixing between $\phi_{0}$ and the CFT states can not any more be considered a small perturbation. This can also be understood from our gravity-side discussion in section 3. Indeed, we are dealing with a situation where the UV-brane mass term is tuned to allow for an exact zero mode. The bulk profile of this zero-mode is $\psi_{0} \sim z^{\frac{1}{2}-\alpha}$. Thus, its normalization is UV-localized 
for $\alpha>1$ and IR-localized for $\alpha<1$ (cf. eq. (3.9)). The corresponding $4 \mathrm{~d}$ field can then be viewed as a UV-brane- or IR-brane-localized field respectively. In the case $\alpha>1$, this supports our previous statement that $\phi_{0}$ does not affect the CFT dynamics significantly. By contrast, in the $\alpha<1$ case, the zero mode is tangled up in a non-trivial way in the non-perturbative CFT dynamics [57-59].

In order to understand the case $\alpha<1$ with tuned $\lambda=\lambda_{0}$ from the CFT perspective, we will first describe the analysis of this section from an equivalent but technically slightly different point of view. When running the Lagrangian of eq. (5.3) down to smaller scales, we are consecutively integrating out $\phi_{0}$ modes with lower and lower 4 -momenta $k$. This induces corrections which are schematically of the form

$$
\sim \frac{1}{\Lambda_{\mathrm{UV}}^{2(\Delta-3)}} \frac{\mathcal{O}_{\Delta}(k)^{2}}{M_{\phi_{0}}^{2}(k)+c(k) k^{2}} .
$$

Here the function $c$ is zero at the high scale, $c\left(\Lambda_{\mathrm{UV}}\right)=0$, and grows at lower scales to the extent that a kinetic term for $\phi_{0}$ is induced by CFT loops. If $M_{\phi_{0}}^{2}(k)$ becomes small and the induced operator $\sim \mathcal{O}_{\Delta}{ }^{2}$ is not suppressed by $\Lambda_{\mathrm{UV}}$ (i.e. for $\Delta<3$ ), it can be large enough to modify the CFT dynamics significantly and to invalidate the derived decay rate. Moreover, the effect of this operator must be the origin of the tachyonic instability which is present in the CFT for $\lambda<\lambda_{0}$.

Alternatively, we may immediately integrate out $\phi_{0}$, before considering any RG running or loop effects. This induces a coupling of the type given in eq. (5.4) (but with $M_{\phi_{0}}^{2}=$ $\left.M_{\phi_{0}}^{2}\left(\Lambda_{\mathrm{UV}}\right)\right)$. It also induces the term

$$
\sim \frac{\mathcal{O}_{\Delta}^{2}}{\left(\Lambda_{\mathrm{UV}}\right)^{2(\Delta-3)} M_{\phi_{0}}^{2}}
$$

which, in contrast to the non-local corrections given schematically in eq. (5.7), is a local operator. In other words, the presence of a UV brane with a brane-localized mass term can be viewed, on the CFT side, as a correction by a 'double trace operator' as discussed in [61].

It is now immediately clear that, as $M_{\phi_{0}}^{2}$ is large in the detuned case, this $\mathcal{O}_{\Delta}^{2}$ correction is too small to affect the CFT dynamics at low energy scales (and in particular the dimension of $\mathcal{O}_{\Delta}$ ) significantly. Hence, the coupling $\mathcal{O}_{\Delta} F^{2}$ can be directly used to estimate the decay rate of any light glueball with non-vanishing overlap with $\mathcal{O}_{\Delta}$. This reproduces eq. (5.5).

It is also clear that, from this perspective, the value $\Delta=2$ (corresponding to $\alpha=0$ ) deserves special attention. Indeed, in this case the $\mathcal{O}_{\Delta}^{2}$ operator is marginal rather than irrelevant. As discussed in section 4 of [61], this causes multiplicative renormalization of the operator $\mathcal{O}_{\Delta}$ with logarithmic running:

$$
\mathcal{O}_{\Delta}\left(m_{\mathrm{IR}}\right) \sim \ln \left(\Lambda_{\mathrm{UV}} / m_{\mathrm{IR}}\right) \mathcal{O}_{\Delta}\left(\Lambda_{\mathrm{UV}}\right) .
$$

Upon rescaling the operator in eq. (5.4), we obtain an additional factor of $\ln \left(\Lambda_{\mathrm{UV}} / m_{\mathrm{IR}}\right)^{-2}$ in the decay rate eq. (5.5), in agreement with the factor $\ln \left(k / m_{\mathrm{IR}}\right)^{-2}$ in the second line of eq. (3.14).

We now consider the case $\alpha<1$ with tuned boundary mass, $\lambda=\lambda_{0}$, corresponding to the largest consistent coefficient of the $\mathcal{O}_{\Delta}^{2}$ correction. From the discussion in section 3 
(see in particular eqs. (3.7) and (3.18)) we see that, in this case, the UV behaviour of the AdS scalar changes from $\Phi \propto z^{2+\alpha}$ to $\Phi \propto z^{2-\alpha}$. According to the general discussion of [53], the dual description of an AdS field theory with such boundary conditions and with $\alpha<1$ is provided by a CFT with an operator $\mathcal{O}_{\Delta^{\prime}}$ with dimension $\Delta^{\prime}=2-\alpha$ (instead of $\Delta=2+\alpha)$. When we integrate $\phi_{0}$ out at the UV scale, we obtain the coupling

$$
\sim \frac{1}{\Lambda_{\mathrm{UV}}^{\Delta^{\prime}}} \mathcal{O}_{\Delta^{\prime}} \operatorname{tr}\left(F^{\mu \nu} F_{\mu \nu}\right)
$$

as well as a term of the type given in eq. (5.8). One may in fact say that it is this latter correction with an appropriately tuned coefficient which forces the dimension of $\mathcal{O}$ to change to $\Delta^{\prime}=4-\Delta$ in the infrared [39] (see also [61, 62]). From eq. (5.10), we can estimate the decay rate of glueballs with non-vanishing overlap with $\mathcal{O}_{\Delta^{\prime}}$ as

$$
\Gamma \sim\left(\frac{m_{\mathrm{IR}}}{\Lambda_{\mathrm{UV}}}\right)^{2 \Delta^{\prime}} m_{\mathrm{IR}},
$$

in agreement with eq. (3.15).

While a more systematic and quantitative study of the CFT description of the decay rates under consideration may be worthwhile, we believe that we have now supplied enough additional physical intuition for the purposes of the present paper. In particular, we have fully confirmed the results of our gravity-side calculation.

\section{Application to throats in flux compactifications}

We now discuss the applicability of our results to throat geometries in type IIB string theory. For definiteness, we focus on the Klebanov-Strassler (KS) throat [8, 60, 63, 64] in the following. We expect, however, that our results can similarly be applied to other types of throats.

The curvature scale $R=k^{-1}$ of the KS throat varies logarithmically along the radial direction and the geometry smoothly terminates in the IR. We neglect this logarithmic variation in the following. Sufficiently far away from the IR tip, the KS throat can then be approximated by the space $\mathrm{AdS}_{5} \times T^{1,1}$. In the UV, the throat is smoothly glued into a compact manifold [1]. We focus on compactifications in which the size $L$ of this manifold is not hierarchically larger than the AdS scale $R$ of the throat. ${ }^{15}$ We can then neglect the 'thickness' of the compact manifold and approximate it by the UV brane of an RS model. Similarly, we model the IR end of the throat by an IR brane (see ref. [9] for more details on the KS throat as a RS model).

The KK reduction of type IIB supergravity on $T^{1,1}$ in an $\mathrm{AdS}_{5} \times T^{1,1}$ background has been carried out in [32-34]. The resulting spectrum of KK modes contains scalars with various tachyonic masses, some of which saturate the Breitenlohner-Freedman (BF) bound. Note that the presence of scalars saturating this bound is expected on general

\footnotetext{
${ }^{15}$ The throat can only be glued smoothly into the compact manifold if $L \gtrsim R$. The distribution of flux vacua strongly favors vacua where the volume $L^{6}$ is small in string units, cf. [65] and references therein. Thus, the generic compactification has no large hierarchy between $R$ and $L$.
} 
grounds: The theory in $\mathrm{AdS}_{5}$, which results from the KK reduction, is supersymmetric. Furthermore, the isometries of $T^{1,1}$ ensure the presence of massless vector multiplets in the spectrum. Such multiplets contain a scalar with maximally tachyonic mass [30, 31, 43].

We will now discuss tachyons from such multiplets in more detail. The KK reduction of type IIB supergravity on $\mathrm{AdS}_{5} \times T^{1,1}$ contains eight massless $5 \mathrm{~d}$ vectors. One of these vectors comes from the compactification of the 4 -form potential on the 3 -cycle in $T^{1,1} \sim$ $S^{3} \times S^{2}$. The corresponding abelian symmetry of the solution is called $\mathrm{U}(1)_{B}$. The seven remaining vectors are associated with the $\mathrm{SO}(4) \times \mathrm{U}(1)_{R}$ isometry of $T^{1,1}$. Since the $\mathrm{U}(1)_{R}$ vector is part of the massless graviton multiplet [32-34] which contains no scalars (and in particular no tachyon), we end up with seven BF tachyons in total. In the classification of [32-34], these scalars belong to shortened versions of vector multiplet I.

In a KS throat, the symmetries are reduced with respect to $\mathrm{AdS}_{5} \times T^{1,1}$. In the region between the IR and UV end, which is the most symmetric part of the throat, the symmetry is reduced to $\mathrm{SO}(4) \times \mathrm{U}(1)_{B} \times \mathbb{Z}_{2 M} \subset \mathrm{SO}(4) \times \mathrm{U}(1)_{B} \times \mathrm{U}(1)_{R}$. The $\mathrm{U}(1)_{R}$ is broken to $\mathbb{Z}_{2 M}$ by the $M$ units of 3 -form-flux on the $S^{3}$, which are also responsible for the logarithmic variation of the AdS scale [66]. Note, however, that this breaking does not affect our previous counting of BF tachyons since, as mentioned before, the $\mathrm{U}(1)_{R}$ vector has no scalar partner.

The symmetry is further reduced at the IR and UV end of the KS throat: In the IR, the $\mathrm{SO}(4)$ stays intact, but the $\mathrm{U}(1)_{B}$ is completely broken, and the $\mathbb{Z}_{2 M}$ is broken to $\mathbb{Z}_{2}[8,67$ 69]. In the UV, on the other hand, the $\mathrm{SO}(4) \times \mathrm{U}(1)_{R}$ isometry of $T^{1,1}$ is broken since a compact Calabi-Yau has no continuous isometries. Concerning the $\mathrm{U}(1)_{R}$ factor, we are anyway only interested in its $\mathbb{Z}_{2}$ subgroup which survives in the IR region. This discrete $\mathbb{Z}_{2}$ symmetry (or a larger discrete subgroup of the $T^{1,1}$ isometry) may or may not be respected by the compact Calabi-Yau. The latter is certainly the generic case. In addition, the KS throat has another $\mathbb{Z}_{2}$ symmetry (called $\mathcal{I}$-symmetry in [69]) which interchanges the two 2-sphere in the $T^{1,1}\left(T^{1,1}\right.$ is an $S^{1}$ bundle over $\left.S^{2} \times S^{2}\right)$ and reverses the sign of the 2-form potentials of type IIB supergravity [60,70]. This symmetry is unbroken in the IR and may or may not be broken in the UV. ${ }^{16}$ Furthermore, the $\mathrm{U}(1)_{B}$ can be broken or remain unbroken in the UV. This is not essential for us since the corresponding BF scalar, being in the adjoint, is uncharged. Thus, the couplings of this scalar to various UV localized fields, which are our main interest, are not forbidden by this symmetry. We finally note that the symmetry breaking in the UV is mediated to the IR by irrelevant operators and is thus suppressed by powers of the warp factor $[15,16,73,74] .^{17}$

We now discuss specifically the effects of the tachyon in the $\mathrm{U}(1)_{B}$ vector multiplet (also known as Betti multiplet). This tachyon is an $\mathrm{SO}(4)$ singlet, but odd under the $\mathcal{I}$-symmetry

\footnotetext{
${ }^{16}$ As long as the throat is infinite, we can move along a one-parameter family of solutions, the so-called baryonic branch of the dual gauge theory, and the KS solution is a special point which respects the $\mathcal{I}$ symmetry $[68,69,71,72]$. However, the metric deformation which corresponds to moving along the baryonic branch does not respect the conformal-Calabi-Yau condition [71]. Hence, it is not clear how to glue such a deformed throat into a compact UV space in the framework of warped flux compactifications [1]. For this reason, we do not consider such deformations. It is, of course, nevertheless possible that the $\mathcal{I}$-symmetry is broken by the Calabi-Yau (instead of the throat), corresponding to a $\mathbb{Z}_{2}$-breaking on the UV brane.

${ }^{17}$ As discussed in [87], relevant perturbations (which grow towards the IR) are also possible as long as they start with a sufficiently small amplitude in the UV.
} 
$\mathbb{Z}_{2}[68] .{ }^{18}$ In the IR region of the KS throat, where the approximation as $\operatorname{AdS}_{5} \times T^{1,1}$ becomes unreliable, this tachyon mixes with a scalar of mass $M^{2}=5 k^{2}[75,76]$. In the UV, on the other hand, the two scalar fluctuations decouple, as we will now demonstrate. The equations of motion of this system are given in eqs. (47) and (48) in [75]:

$$
\begin{aligned}
\tilde{z}^{\prime \prime}-\frac{2}{\sinh ^{2} \tau} \tilde{z}+\tilde{m}^{2} \frac{I(\tau)}{K^{2}(\tau)} \tilde{z} & =\tilde{m}^{2} \frac{9}{4 \cdot 2^{2 / 3}} K(\tau) \tilde{w} \\
\tilde{w}^{\prime \prime}-\frac{\cosh ^{2} \tau+1}{\sinh ^{2} \tau} \tilde{w}+\tilde{m}^{2} \frac{I(\tau)}{K^{2}(\tau)} \tilde{w} & =\frac{16}{9} K(\tau) \tilde{z} .
\end{aligned}
$$

Here, primes denote derivatives with respect to the radial coordinate $\tau$ of the KS throat (using the metric convention of [8]) and $\tilde{m}$ is related to the $4 \mathrm{~d}$ mass of the state. The functions $I(\tau)$ and $K(\tau)$ are e.g. given in appendix B in [75]. In the UV, $\tau \gg 1$, these functions can be approximated by $I(\tau) \sim \tau e^{-4 \tau / 3}$ and $K(\tau) \sim e^{-\tau / 3}$. The equations of motion then indeed decouple and simplify to

$$
\begin{aligned}
\tilde{z}^{\prime \prime} & =0 \\
\tilde{w}^{\prime \prime}-\tilde{w} & =0 .
\end{aligned}
$$

This is solved by $\tilde{z}=\tau+$ const. and $\tilde{w}=e^{\tau}+$ const. $e^{-\tau}$ (neglecting the overall factors). For $\tau \gg 1$, the coordinate $\tau$ is related to our coordinate $z$ by $z \propto e^{-\tau / 3}$ and the wavefunctions read $\tilde{z}=\ln z+$ const. and $\tilde{w}=z^{-3}+$ const. $z^{3}$. Up to an overall factor of $z^{2}$ (which is related to a field redefinition ${ }^{19}$ ) these are indeed the wavefunctions of a tachyon which saturates the $\mathrm{BF}$ bound and a scalar with mass $M^{2}=5 k^{2}$. In particular, we see that the wavefunctions from the $\mathrm{KK}$ decomposition on $\mathrm{AdS}_{5} \times \mathrm{T}^{1,1}$ are a good approximation in the UV of the KS throat.

The mass spectrum of $4 \mathrm{~d}$ KK modes from this system of two scalars was determined in [75] and found to contain the lightest state which is known so far for the KS throat (see e.g. [77-82] for other parts of the mass spectrum). Since heavier KK modes decay very quickly to lighter states via various processes (which we will discuss in section 7 ), this lightest KK mode generically contains an $\mathcal{O}(1)$-fraction of the energy density of a heated KS throat. ${ }^{20}$ Due to the effect of the $\mathrm{U}(1)_{B}$ tachyon, these particles decay to the UV sector with the rate determined in section 3 .

The approximate $\mathrm{SO}(4)$ symmetry of the IR region suppresses decays violating the total $\mathrm{SO}(4)$ charge. Therefore, a sizeable fraction of the energy density in a KS throat is generically in the form of charged KK modes. We will now argue that tachyons are also

\footnotetext{
${ }^{18}$ We note that, in the infinite throat limit, this $5 \mathrm{~d}$ scalar has a massless $4 \mathrm{~d}$ mode [68, 75]. When the throat is glued into a compact manifold, this mode obtains a mass which is parametrically large compared to the IR scale $m_{\mathrm{IR}}$ if the volume of the compact manifold is small. Thus, this mode is not important in our context.

${ }^{19}$ In particular, the field $\tilde{z}$ is related to the fluctuation $\psi=\delta g_{13}=\delta g_{24}$ of the 5-dimensional compact manifold in the throat by the field redefinition $\tilde{z}=z^{-2} \psi$. In the UV of the throat, where the approximation as $\mathrm{AdS}_{5} \times T^{1,1}$ is applicable, the field $\psi$ is a $5 \mathrm{~d}$ scalar with a standard kinetic term (coming from the $10 \mathrm{~d}$ Einstein-Hilbert term).

${ }^{20}$ More precisely, this state is part of a massive vector multiplet of $4 \mathrm{~d} \mathcal{N}=1$ supersymmetry [76]. A similar fraction of the energy density is therefore stored in the superpartners of this scalar.
} 
relevant for the decay of these states. Namely, the spectrum in [32-34] contains tachyons with various charges, though not all of them have the maximally allowed negative masssquared. For example, the tachyon in the $\mathrm{SO}(4)$ vector multiplet is in the adjoint of $\mathrm{SO}(4)$. We expect that these tachyons mix with other scalars with the same charge in the IR region of the KS throat. Various IR-localized states can therefore decay via a given tachyon to the UV sector, and the decay rates from section 3 apply. As another example, we can consider KK modes which are dual to glueballs created by the operator of lowest dimension, $\Delta=\frac{3}{2}$, in the KS theory. These states are scalars in the $\left(\frac{1}{2}, \frac{1}{2}\right)$ of $\mathrm{SU}(2) \times \mathrm{SU}(2) \sim \mathrm{SO}(4)$. In [76], the lightest KK mode in this tower was proposed as a candidate for the lightest state in the KS spectrum. A scalar operator of dimension $\Delta=\frac{3}{2}$ is dual to a tachyon with $\alpha=2-\Delta=\frac{1}{2}$ (cf. section 5 ). The decay rate of the corresponding KK modes then follows from the formulas in section 3 .

We now discuss the UV-brane mass term of the various tachyonic scalars. As emphasized before, we focus on compactifications where all moduli are stabilized by fluxes and non-perturbative effects along the lines of $[1,10]$. Let us first assume for simplicity that both complex structure and Kähler moduli are stabilized at the UV scale. In 5d language this means that the UV brane theory has only one energy scale: $k \sim R^{-1} \sim L^{-1} \sim M_{\text {string. }}$. (For simplicity, we ignore the hierarchy between $L^{-1}$ and $M_{\text {string, }}$, assuming it to be small.) Since the full construction is stable, we know that all tachyonic scalars will receive a UVbrane mass term with $\lambda \geq \lambda_{0}$ in this context. Given that there is no small energy scale around (which could correspond to $\lambda-\lambda_{0} \ll 1$ ) and no obvious reason for the tuning $\lambda=\lambda_{0}$, we make the assumption that $\lambda=\lambda_{0}+\mathcal{O}(1)$. As we will see in section 7 , even with this conservative assumption the cosmological effects of tachyonic scalars can be dramatic.

There are, however, at least two possible loopholes in this conclusion. The first loophole is related to the question where the massless mode of a $5 \mathrm{~d}$ tachyon with tuned UV-brane mass is localized. To see this, let us first strengthen our previous argument for detuning in the following way: Recall that the wavefunction of the massless mode for supersymmetric boundary conditions, $\psi \propto z^{\frac{1}{2}-\alpha}$, is UV-localized for $\alpha>1$ (cf. sections 3.2 and 5 ). Therefore, even if we change the boundary condition at the IR brane (e.g. by choosing a different boundary mass term), this mode only picks up an exponentially small mass. It is then clear that, for tuned UV mass term and $\alpha>1$, there is always an exponentially light UV-localized mode in the spectrum. However, with all Calabi-Yau moduli stabilized at the high scale, there should, in 5d language, be no light fields at or near the UV brane. Thus, the UV-localized mode can not be light, implying that the UV mass term has to be detuned. For $\alpha<1$, however, this argument does not apply as the wavefunction is IR-localized in this case. The zero mode of such a tachyon can be light without violating the requirement that there are no light fields in the UV sector. Furthermore, for generic IR-boundary condition, this mode picks up a mass of the order the IR scale. Thus, for $\alpha<1$, the UV mass term can in principle be tuned. Of course, we still have no reason for the required tuning, but we can also not dismiss the tuned situation on general grounds.

The second loophole lies in our assumption that there are no light fields in the UV sector. There is obviously the possibility that the Kähler stabilization scale is much smaller than the flux stabilization scale, which may be the natural choice for low-scale SUSY. In 
this case, the UV-brane theory has a second, smaller energy scale with corresponding light fields. In the $5 \mathrm{~d}$ description, these fields could be the lowest KK modes of tachyons. More precisely, for tuned $\lambda=\lambda_{0}$ and $\alpha>1$, such a mode is massless and localized in the UV, corresponding to an unstabilized modulus. Stabilization of the Kähler moduli, in the 5d description, is then due to a detuning of the mass on the UV brane. If the Kähler stabilization scale is lower than the flux stabilization scale (which we assume to be of the order the AdS scale), this detuning is small, $\lambda-\lambda_{0} \ll 1$, giving the modulus a small mass compared to the AdS scale. Of course, as far as some generic Kähler modulus is concerned, such a connection is far from obvious because the throat has no 2- or 4-cycles. ${ }^{21}$ However, such an approximate tuning can potentially be related to the universal Kähler modulus. ${ }^{22}$ If the detuning is sufficiently small, the higher KK modes of the corresponding tachyon would decay with the enhanced rates derived in section 3.2 .

In summary, we have very good reasons to consider decays mediated by tachyons in throat cosmology. While the situation with detuned UV mass term appears to be generic, we can not exclude the possibility that certain tachyonic fields have a UV mass tuned to the minimal allowed value. Establishing this, which would imply even larger decay rates than we discuss in the following, would be an interesting subject for future research.

Finally, we note a recent paper [19] which presented a survey of decay channels of KK modes in a KS throat. In particular, the decay rate of KK modes to moduli and their axionic partners was determined. As the moduli can be viewed (at least partially) as fields living on the UV brane, it is interesting to compare this decay rate with our results. More precisely, eq. (5.5) in [19] gives the decay rate of a KK mode into axionic partners of the moduli. ${ }^{23}$ For $M_{p} \sim M_{s} \sim k$ and using our notation $w=m_{\mathrm{IR}} / k$ and $\nu^{\star}=\alpha$, their eq. (5.5) reads

$$
\Gamma \sim\left(\frac{m_{\mathrm{IR}}}{k}\right)^{4+2 \alpha} m_{\mathrm{IR}}
$$

This agrees with our eq. (3.14) (apart from the logarithmic suppression in the case $\alpha=0$ ). However, we believe that this rate also applies to the tunneling of KK modes to other throats. As discussed in [46] in some detail, via the AdS/CFT correspondence, other throats can be described by large- $N$ gauge theories which live on the UV brane. The tunneling rate of KK modes to these sectors is then given by eq. (6.5) times the number of degrees of freedom, $\sim N^{2}$, of the dual gauge theories.

\footnotetext{
${ }^{21}$ Note that $T^{1,1}$ is isomorphic to $S^{2} \times S^{3}$ and thus has a nontrivial 2-cycle [60]. However, this $S^{2}$ shrinks to zero at the IR tip of the throat and thus is not a cycle of the Calabi-Yau.

${ }^{22}$ It is clear that the universal Kähler modulus belongs to the UV sector if we can work in a Kähler-Weyl frame (the Brans-Dicke frame). In this frame, the metric in the IR region is not affected by a (small) shift of the universal Kähler modulus. Namely, such a shift corresponds to changing the prefactor of the 4d Einstein-Hilbert term, which is dominated by the compact Calabi-Yau and the UV end of the throat. However, it has recently been argued that this is not always the appropriate frame [83, 84]. In other frames, the universal Kähler modulus may be viewed (at least partially) as a throat field.

${ }^{23}$ Note that the decay rate in their eq. (5.4) is additionally suppressed as it involves a transition between states with different charges under the approximately conserved symmetries of the throat.
} 


\section{Cosmology}

To illustrate the relevance of tachyons, we will now discuss the reheating of the Standard Model (SM $)^{24}$ after warped brane inflation. For definiteness, we focus on a specific realization of warped brane inflation, the KKLMMT scenario [11]. In this scenario, inflation is driven by a D3-brane which slowly rolls towards an anti-D3-brane located at the tip of a KS throat. As discussed in appendix C of [11], for given parameters (string coupling, string scale, etc.), the hierarchy of this throat can be fixed by observational data. ${ }^{25}$ In the following, we will use $m_{\mathrm{IR}} \sim 10^{14} \mathrm{GeV}$ and $k \sim 10^{18} \mathrm{GeV}$ for numerical estimates. We assume that the SM is realized on D-branes which are localized in the CY outside the throat. In the effective $5 \mathrm{~d}$ description, the SM then lives on the UV brane of the corresponding RS model. Furthermore, we assume that the inflationary throat is the only strongly warped region in the compact space.

Inflation ends with the annihilation of the D3-brane and the anti-D3-brane. The resulting energy is deposited in KK modes localized at the tip of the throat [20-23, 85]. Temperature and energy density of this gas, which is initially marginally non-relativistic, are set by the IR scale: $T \sim m_{\mathrm{IR}}$ and $\rho \sim m_{\mathrm{IR}}^{4}$. These KK modes decay to the SM at later times. The resulting reheating temperature of the SM can be estimated as usual: Most of the energy is transferred when the Hubble rate is comparable to the decay rate. The Hubble rate is related to the total energy density by $H^{2}=\rho_{\text {tot }} / 3 M_{4}^{2}$. For radiation at temperature $T$ and with $g_{\mathrm{SM}}$ degrees of freedom, the energy density is given by $\rho_{\text {tot }}=\frac{\pi^{2}}{30} g_{\mathrm{SM}} T^{4}$. The reheating temperature can then be estimated as

$$
T_{\mathrm{RH}} \sim\left(\frac{10}{g_{\mathrm{SM}}}\right)^{\frac{1}{4}} \sqrt{M_{4} \Gamma} .
$$

It is probable that the energy density in the throat is in the form of different species of KK modes with different lifetimes. After one species of KK modes has decayed to the SM, KK modes with longer lifetimes can easily come to dominate the total energy density as their energy density scales like matter (and not like radiation). Their decay to the SM then leads to a new phase of reheating and the final reheating temperature will be determined by the most stable KK modes. Let us now assume that the throat has no tachyonic scalars. There certainly are scalar KK modes in the spectrum of the KS throat (e.g. from the dilaton). Without tachyons, their largest possible decay rate would be that for a massless scalar, corresponding to $\alpha=2$. As discussed in section 6 , we expect that scalars generically obtain a mass on the UV brane (in the 5 d description) and that this mass is not tuned to the special value $\lambda_{0}$. Using the corresponding decay rate for a massless scalar, eq. (3.14), we

\footnotetext{
${ }^{24}$ We use the term 'Standard Model' to refer to a sector which contains the SM, e.g. the MSSM. For definiteness, we use $g_{\mathrm{SM}}=\mathcal{O}(100)$ for the number of effective relativistic degrees of freedom at the reheating temperature $T_{\mathrm{RH}}$. For example, the SM itself has $g_{\mathrm{SM}} \approx 107$ for temperatures $T \gtrsim 300 \mathrm{GeV}$ while the MSSM has $g_{\mathrm{SM}} \approx 229$ for $T \gtrsim m_{1 / 2}$ (with the familiar prefactor $7 / 8$ for fermions included).

${ }^{25}$ Some fine-tuning is generically required to actually achieve slow roll inflation. A systematic study was carried out in [87] (see also [88, 91] for earlier work). Some of the mechanisms used in these papers rely on D7-branes wrapping 3-cycles of the throat (see e.g. [92] for an explicit realization). For our purposes, we assume a proper fine-tuning such that a KKLMMT-type scenario is realized.
} 
then obtain an upper bound on the reheating temperature in a scenario without tachyons:

$$
T_{\mathrm{RH}} \lesssim 1 \mathrm{GeV}
$$

This is dangerously low. In particular, it is difficult to obtain sufficient baryogenesis at such low temperatures. Moreover, reheating temperatures below $1 \mathrm{MeV}$ are excluded by nucleosynthesis [95-97]. As tachyons lead to higher decay rates, the reheating temperature can also be higher. We will now discuss this in more detail.

We first consider processes in the throat sector which happen on timescales shorter than those relevant for decays to the SM. Warped KK modes are described by an effective field theory with a low cutoff, $\sim m_{\mathrm{IR}}$. We do not assume a large hierarchy between $k$ and $M_{5}$ (or, from the $10 \mathrm{~d}$ perspective, $k, M_{s}$ and $M_{10}$ ) which would suppress quantum corrections. All possible n-point-interactions, which are allowed by the symmetries, will therefore be induced by quantum effects (if they are not present at tree-level). In particular, the effective action includes couplings of the type

$$
\mathcal{H} \mathcal{H} \mathcal{G} \text {, }
$$

which involves two different species of KK modes $\mathcal{H}$ and $\mathcal{G}$ and allows for the processes $2 \cdot \mathcal{H} \leftrightarrow 2 \cdot \mathcal{G}$. Here, we have suppressed all symmetry-indices (which are appropriately contracted). Let $\mathcal{G}$ denote the lightest $\mathrm{KK}$ mode in the spectrum, whereas $\mathcal{H}$ is any heavier KK mode. As we will see in a moment, the KK modes are in thermal equilibrium initially. For temperatures below the mass of the heavier KK mode, the process $2 \cdot \mathcal{H} \rightarrow 2 \cdot \mathcal{G}$ then occurs with a much higher rate than the inverse process. The heavier KK modes thus begin to annihilate to the lighter KK modes, leading to an exponential decrease of the relative number density of the two states,

$$
\frac{n_{\mathcal{H}}}{n_{\mathcal{G}}}=e^{-\left(m_{\mathcal{H}}-m_{\mathcal{G}}\right) / T} .
$$

Here, $m_{\mathcal{H}}$ and $m_{\mathcal{G}}$ are the masses of the two states and $T$ is the temperature of the gas of KK modes. This exponential decrease continues until the heavier KK modes are so dilute that they decouple. This happens, when

$$
n_{\mathcal{H}} \cdot\langle\sigma v\rangle \sim H
$$

where $\langle\sigma v\rangle$ is the thermally averaged product of cross section and relative velocity for the scattering process and $H$ is the Hubble rate. The latter is dominated by the energy density $\rho$ of the gas of KK modes, $H \sim \sqrt{\rho} / M_{4}$. Due to the exponential dependence on the temperature in eq. (7.4), the heavy KK modes decouple when the temperature is still of the order $m_{\mathrm{IR}} \cdot{ }^{26}$ By dimensional analysis, it then follows that $\langle\sigma v\rangle \sim m_{\mathrm{IR}}^{-2}$ as well as $\rho \sim m_{\mathrm{IR}}^{4}$ and $n_{\mathcal{G}} \sim m_{\mathrm{IR}}^{3}$. Using eq. (7.5), we find

$$
\frac{n_{\mathcal{H}}}{n_{\mathcal{G}}} \sim \frac{m_{\mathrm{IR}}}{M_{4}} \sim 10^{-4}
$$

\footnotetext{
${ }^{26}$ More precisely, the condition on the decoupling temperature $T_{\mathrm{dec}}$ follows from eqs. (7.4) and (7.5).

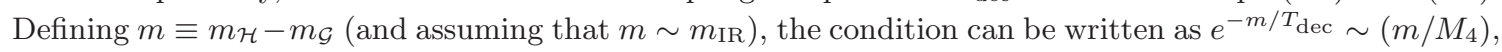
where we have neglected powers of $m / T_{\text {dec }}$ multiplying the right-hand side. For $m_{\mathrm{IR}} / M_{4} \sim 10^{-4}$, we then find that $T_{\mathrm{dec}} \sim m_{\mathrm{IR}} / 9$.
} 
The fact that this factor is smaller than 1 shows that the KK modes were indeed in thermal equilibrium initially. Thus, the energy density is dominantly in the form of the lightest KK mode. All heavier KK modes are $10^{-4}$ times less abundant.

There are other decay processes in the throat sector. In particular, the effective action will also contain various trilinear couplings. If kinematically allowed, KK modes can therefore decay to two lighter KK modes. If these processes are not suppressed due to the approximate symmetry $\mathrm{SO}(4) \times \mathbb{Z}_{2} \times \mathbb{Z}_{2}$ of the effective action (cf. section 6 ), the corresponding decay rate is $\sim m_{\mathrm{IR}}$ (as follows by dimensional analysis). Moreover, we expect various couplings of $\mathrm{KK}$ modes to $4 \mathrm{~d}$ gravitons in the effective action. While such couplings are forbidden by KK-mode orthogonality at the level of the two-derivative, quadratic action $[14,16]$, they are certainly present in the strongly-coupled and highly non-linear effective field theory relevant for the dynamics of the low-lying KK modes.

Indeed, integrating out the heavier states, all types of multi-particle and higherderivative vertices are generated in the effective action for the lowest-lying KK modes. Since any derivatives appearing in this action are covariant, various quantities derived from the Riemann tensor naturally arise. As we assume no significant hierarchy between the AdS scale and the Planck scale, the only scale relevant for this argument is the IR scale $m_{\mathrm{IR}}$. Thus, for example, we naturally expect a term of the type

$$
\sim \frac{1}{m_{\mathrm{IR}}} R^{2} \mathcal{G}
$$

to arise. Here, $R^{2}$ is some scalar quadratic in the $4 \mathrm{~d}$ Riemann tensor and the scalar KK mode $\mathcal{G}$ is a singlet with respect to the symmetries of the effective action (otherwise, the term would be forbidden or suppressed). We therefore believe that such terms are generically present in the effective action and that they are not suppressed by powers of the warp factor, a possibility raised in [19]. As discussed in [19, 93, 94], the coupling eq. (7.7) allows for the decay of $\mathcal{G}$ to two $4 \mathrm{~d}$ gravitons. By canonically normalizing the gravitons, the relevant vertex is seen to be suppressed by $1 / M_{4}^{2}$, leading to the decay rate $\sim m_{\mathrm{IR}}^{5} / M_{4}^{4}$. Similar arguments can be made for couplings involving two KK modes and a graviton. Heavier KK modes can therefore decay to lighter KK modes via the emission of a graviton. If these processes are not suppressed due to the approximate symmetries of the effective action, the corresponding decay rate is $\sim m_{\mathrm{IR}}^{3} / M_{4}^{2}$.

Let us summarize what we have found: At late times, the energy density is dominantly in the form of the lightest KK mode. As we have discussed in section 6, the lightest known KK mode in the KS throat mixes with the tachyon from the Betti multiplet. We will assume that this KK mode is indeed the lightest state. It thus decays to the SM with the rate for a maximally tachyonic scalar:

$$
\Gamma \sim\left(\frac{m_{\mathrm{IR}}}{k}\right)^{4} \frac{m_{\mathrm{IR}}}{\ln ^{2}\left(k / m_{\mathrm{IR}}\right)} .
$$

As the lightest known KK mode is odd with respect to the $\mathcal{I}$-symmetry (cf. section 6 ), the decay to two gravitons is forbidden or strongly suppressed (compared to the rate $\sim m_{\mathrm{IR}}^{5} / k^{4}$ for $k \sim M_{4}$ ). Thus, the decay to the SM is the dominant decay channel for this state. 
The heavier KK modes are $10^{-4}$ times less abundant than the lightest KK mode. In addition, several of these states decay to lighter KK modes via trilinear couplings and graviton emission. The timescales for these decays are $\sim m_{\mathrm{IR}}^{-1}$ and $\sim M_{4}^{2} / m_{\mathrm{IR}}^{3}$, respectively. At later times, only the lightest $\mathrm{KK}$ modes with given charges under $\mathrm{SO}(4) \times \mathbb{Z}_{2} \times \mathbb{Z}_{2}$ may survive. Moreover, as this symmetry is only approximate, eventually all KK modes can decay to the lightest KK mode. Such charge violating decays are, however, suppressed by additional powers of the warp factor $[15,16,73,74]$. If the decay rate is nevertheless higher than eq. (7.8), all KK modes will first decay to the lightest KK mode. The latter subsequently decay to the SM. Using eq. (7.1), the resulting reheating temperature of the SM is

$$
T_{\mathrm{RH}} \sim 10^{7} \mathrm{GeV} .
$$

This is considerably higher than $1 \mathrm{GeV}$, due to the enhanced decay rate mediated by a tachyon. It is also possible, however, that the total decay rate of the heavier KK modes (to the SM and to the lightest KK mode) is smaller than eq. (7.8). These states will then still be stable when the lightest KK mode has already decayed to the SM. As their energy density scales like matter whereas the energy density of the SM scales like radiation, the heavier KK modes might come to dominate the total energy density. Their decay to the SM then leads to a new phase of reheating with a lower reheating temperature. Although we have not analysed this possibility in more detail, it is clear that tachyons may also enhance the relevant decay rates (and thus the reheating temperature) in this case.

Finally, we briefly consider the decay of fermionic KK modes. If supersymmetry is broken outside the throat, we expect that the fermionic decay rates are of the same order of magnitude as those of the scalars in the same multiplet. However, the decays of fermions are somewhat model-dependent as the supersymmetry breaking scale (or the gravitino mass $m_{3 / 2}$ ) determines the allowed decay channels. If supersymmetry is broken at low scales, $m_{3 / 2}<m_{\mathrm{IR}},{ }^{27}$ the fermionic KK modes can decay to lighter KK modes under the emission of a gravitino. Another possible channel is the decay to a graviton and a gravitino. These processes are the analogue of the decay of bosonic KK modes to gravitons. The resulting abundance of gravitinos leads to the well-known gravitino problem. If the supersymmetry breaking scale is high, $m_{3 / 2}>m_{\mathrm{IR}}$, decays to gravitinos and decays to superpartners of standard model particles are kinematically forbidden. As we have assumed that the inflationary throat is the only strongly warped region (and the fermionic KK modes can thus not decay to such sectors), the fermionic KK modes may then be absolutely stable. In order to avoid the resulting overclosure of the universe, one can invoke the neutrino portal which allows their decay to a neutrino and a Higgs [17, 101].

\section{Conclusions}

In this paper, we have analysed the effect of tachyonic scalars on couplings between IR- and UV-localized sectors in warped compactifications. We gave an introduction to tachyonic

\footnotetext{
${ }^{27}$ In some scenarios of brane inflation the gravitino mass provides an upper bound on the inflationary Hubble scale, $H_{I} \lesssim m_{3 / 2}[98,99]$. Since $H_{I} \sim m_{\mathrm{IR}}^{2} / M_{4}$, the case $m_{3 / 2}<m_{\mathrm{IR}}$ is possible also in these scenarios.
} 
scalars in a slice of $\mathrm{AdS}_{5}$ in section 2. In particular, we explained the origin of an instability and its removal via a UV-brane-localized mass term for the field, both of which can be easily understood in a quantum mechanical analogue. In section 3 we considered decays of IR-localized KK-modes to a UV-localized gauge theory in a 5d Randall-Sundrum toy model and derived the dependence of the decay rates on the warp factor. As expected, this dependence is governed by the $5 \mathrm{~d}$ mass of the scalar. There is also a further suppression due to the UV-localized mass term.

We have developed the dual CFT description of our results in section 5. In this approach, the dependence on the $5 \mathrm{~d}$ mass $M$ is encoded in the dimension of the dual operator, given by $\Delta=2+\sqrt{4+M^{2} / k^{2}}$. As long as the UV-brane mass term takes generic values above the boundary for stability, its effect on decay rates can be understood as a simple propagator suppression on the CFT side. If this boundary mass term is tuned to its minimal allowed value, decay rates are correspondingly enhanced. While this is straightforward to see for $\Delta>3$, the analysis of such a tuned situation in the regime with $\Delta<3$ is more subtle: Here, one has to use the alternative CFT description with an operator of dimension $\Delta^{\prime}=4-\Delta$.

In section 6 , we have worked out the applicability of our $5 \mathrm{~d}$ analysis to throat geometries in string compactifications. As a specific example, we have considered the KlebanovStrassler throat with its approximate $\mathrm{AdS}_{5} \times T^{1,1}$ geometry in the UV region. Due to the (gauge) symmetries of the solution, tachyonic $5 \mathrm{~d}$ scalars saturating the BreitenlohnerFreedman bound are present. We have argued that, generically, the UV-brane mass of these tachyons is detuned from its minimal value. ${ }^{28}$ The spectrum of light KK modes in the Klebanov-Strassler throat has been studied (cf. e.g. [75-82]) and the lightest state found so far is a singlet under the continuous symmetries of the throat. This state results from the mixture of two $5 \mathrm{~d}$ scalars, one of which has maximally tachyonic $5 \mathrm{~d}$ mass. Using the equations of motion of the two coupled scalars, we have checked that the two fluctuations decouple in the UV and that one of the resulting wavefunctions correctly describes a maximally tachyonic scalar. The lightest (known) KK mode of the Klebanov-Strassler throat thus decays to the UV brane with the decay rate derived in section 3 .

In section 7, we have used a specific example, the reheating of the standard model after warped brane inflation, to demonstrate the relevance of tachyons in throat cosmology. Since tachyons affect the decay rate to the UV brane, their role is decisive in settings where the SM is localized outside the inflationary throat. We have focused on such scenarios assuming, for definiteness, that the SM is realized in the bulk of the Calabi-Yau. ${ }^{29}$ An important consequence of tachyons is that they lead to a larger reheating temperature of the standard model. More precisely, without tachyons, one finds an upper bound on the reheating temperature $T_{\mathrm{RH}} \lesssim 1 \mathrm{GeV}$. Including the tachyons, one instead has $T_{\mathrm{RH}} \sim$

\footnotetext{
${ }^{28}$ We have, however, also pointed out potential loopholes in this argument. Thus, the case of a tuned UV mass term can not be completely dismissed. Such a tuning would allow for decay rates which are even higher than those which we found in the generic situation.

${ }^{29}$ We believe, however, that our results also apply to situations where the SM lives in another throat. In this case, our decay rates have to be multiplied by the number of degrees of freedom, $\sim N^{2}$, of the large- $N$ gauge theory which is dual to the additional throat.
} 
$10^{7} \mathrm{GeV}$. Charged KK modes may have smaller decay rates and correspondingly larger lifetimes. Their decay may lead to another phase of reheating, resulting in a lower reheating temperature. Although we have not determined the decay rate of a generic charged KK mode, it is likely that their decay rates are also enhanced by tachyons.

To discuss this in more detail, we recall that the UV region of the Klebanov-Strassler throat can be approximated as $\mathrm{AdS}_{5} \times T^{1,1}$. In this region, the $\mathrm{KK}$ reduction on $T^{1,1}$, which was performed in [32-34], is applicable. Close to the IR end of the throat, the approximation as $\mathrm{AdS}_{5} \times T^{1,1}$ becomes unreliable. We expect that a given $\mathrm{AdS}_{5}$ field (coming from the KK reduction on $T^{1,1}$ ) mixes with all other fields with the same quantum numbers in this region. This is likely already the case at tree-level as follows e.g. from the analysis in [75, 76, 81]. Moreover, there generically is no large hierarchy between the 10-dimensional Planck scale and the AdS curvature scale which could strongly suppress quantum corrections. Thus, we expect all bilinear mixing terms, which are allowed by the symmetries, to appear in the effective $5 \mathrm{~d}$ action. A $4 \mathrm{~d}$ KK mode with given quantum numbers will therefore couple to the UV-brane via all $\mathrm{AdS}_{5}$ fields with the same quantum numbers. It is then clear that the decay rate of a scalar KK mode will be determined by the $\mathrm{AdS}_{5}$ field with the smallest (or possibly most tachyonic) mass-squared for the given charges. As the spectrum of these fields is known [32-34], it should be possible to determine the decay rate of a generic scalar KK mode from its charges under the (approximate) symmetries of the throat. This would be an interesting project for future research.

Another important cosmological application of our results is to throat dark matter [1419, 21]. Let us first consider scenarios in which the standard model is localized outside the throat. Measurements of the cosmic diffuse $\gamma$-ray background give a lower bound of $\sim 10^{26} \mathrm{~s}$ for the lifetime of dark matter with decay channels to photons (assuming an $\mathcal{O}(1$ ) branching ratio for decays via hadrons) [100]. As we have discussed in section 6 , the lightest known KK mode of the Klebanov-Strassler throat couples to the UV-brane via a maximally tachyonic scalar. Generically, a considerable fraction of the dark matter will be in the form of this lightest state. In order to fulfill the above bound, the dark matter throat then has to have an IR scale which is smaller than $\sim 10^{5} \mathrm{GeV}$ (assuming $k \sim 10^{18} \mathrm{GeV}$ ). This effectively excludes KK modes in an inflationary throat à la KKLMMT [11] as a viable dark matter candidate. Dark matter in sufficiently long throats, which may be produced by tunneling from the inflationary throat $[14,21]$ or thermally from the standard model [17], can however still be viable. It may, of course, turn out that the lightest KK mode of the Klebanov-Strassler throat is not the one considered in section 6. However, the mass of a glueball can roughly be expected to decrease with the dimension of the operator which creates the state $[75,76]$. Or, correspondingly, the mass of KK modes generically decreases with the mass of the corresponding $\mathrm{AdS}_{5}$ field [15]. It is therefore likely that any lighter KK mode is also associated with a $5 \mathrm{~d}$ tachyon which determines its decay rate. Finally, dark matter can be formed by fermionic KK modes which can decay to the standard model via the neutrino portal $[17,101]$. The decay rate is likely to be determined by the superpartner of a tachyonic $5 \mathrm{~d}$ scalar and can be suppressed by (approximate) R-parity.

Alternatively, in scenarios in which the standard model is located in the dark matter throat $[14-16,19,21]$, our results are relevant for the decay of dark matter to other throats 
(which are likely to be present in a given compactification [7]). If the dark matter consists of the lightest (known) KK mode, ${ }^{30}$ the requirement that the dark matter lives longer than the current age of the universe gives an upper bound of $\sim 10^{7} \mathrm{GeV}$ on the IR scale of the throat (again assuming $k \sim 10^{18} \mathrm{GeV}$ ). The preferred scale $\sim \mathrm{TeV}$ thus still allows for a viable dark matter candidate (provided it is sufficiently stable against decay to the standard model). As we have discussed above, even if the dark matter instead consists of other charged KK modes, tachyons are again likely to determine their decay rate to the UV sector.

\section{Acknowledgments}

We would like to thank Johanna Erdmenger, Tony Gherghetta, Dam T. Son and Gianmassimo Tasinato for helpful comments and discussions. A.H. is grateful to the Berkeley Center for Theoretical Physics for hospitality. This work was supported by the German Research Foundation (DFG) within the Transregional Collaborative Research Centre TR33 "The Dark Universe".

\section{A Decay rates with Bessel functions}

The purpose of this appendix is to compute the decay rates estimated in section 3 explicitly in terms of Bessel functions. Similar calculations to those below can be found e.g. in [12, $31,102,103]$.

The analytic solution to eq. (3.5) in terms of Bessel functions is given by

$$
\psi_{n}=\frac{1}{a_{\alpha, n}} \widehat{z}^{1 / 2}\left[J_{\alpha}(\widehat{z})+b_{\alpha, n} Y_{\alpha}(\widehat{z})\right],
$$

where $a_{\alpha, n}$ and $b_{\alpha, n}$ are constants determined from the normalization of $\psi_{n}$, eq. (3.9), and the boundary conditions, eq. (3.6).

In order to relate the analytic solution to the previous estimates, we consider the asymptotic expansions of the Bessel functions in the regions of small and large argument [104]. For $0<z \ll \sqrt{\alpha+1}$ :

$$
J_{\alpha}(z) \simeq \frac{1}{\Gamma(\alpha+1)}\left(\frac{z}{2}\right)^{\alpha}
$$

and

$$
Y_{\alpha}(z) \simeq \begin{cases}\frac{2}{\pi}(\gamma-\ln 2+\ln z) & \text { for } \alpha=0 \\ -\frac{\Gamma(\alpha)}{\pi}\left(\frac{2}{z}\right)^{\alpha}-\frac{\cos (\pi \alpha) \Gamma(-\alpha)}{\pi}\left(\frac{z}{2}\right)^{\alpha} & \text { for } \alpha>0\end{cases}
$$

Here, $\gamma \simeq 0.5772$ is the Euler constant and $\Gamma(\alpha)$ is the gamma function. The second term in the expansion for $Y_{\alpha}$ is important in the limit $\alpha \rightarrow 0$. Furthermore, for $z \gg\left|\alpha^{2}-\frac{1}{4}\right|$ :

$$
J_{\alpha}(z) \simeq \sqrt{\frac{2}{\pi z}} \cos \left(z-\frac{\alpha \pi}{2}-\frac{\pi}{4}\right)
$$

\footnotetext{
${ }^{30}$ Note that this state is odd under a $\mathbb{Z}_{2}$-symmetry and may therefore be stable against decay to the standard model.
} 
and

$$
Y_{\alpha}(z) \simeq \sqrt{\frac{2}{\pi z}} \sin \left(z-\frac{\alpha \pi}{2}-\frac{\pi}{4}\right) .
$$

From these expansions it is clear that the Bessel functions explicitly realize the matching of UV and IR solutions discussed in section 3, cf. eqs. (3.7) and (3.8).

Moreover, the Bessel functions satisfy certain identities which are useful in the calculation of the decay rates below. For any linear combination $\mathcal{C}_{\alpha}(z)$ of Bessel functions $J_{\alpha}(z)$ and $Y_{\alpha}(z)$ in which the coefficients are independent of $\alpha$ and $z$, the following identities are valid [104]:

$$
\begin{aligned}
\frac{2 \alpha}{z} \mathcal{C}_{\alpha}(z) & =\mathcal{C}_{\alpha+1}(z)+\mathcal{C}_{\alpha-1}(z) \\
2 \frac{d}{d z} \mathcal{C}_{\alpha}(z) & =\mathcal{C}_{\alpha-1}(z)-\mathcal{C}_{\alpha+1}(z)
\end{aligned}
$$

and

$$
\int_{a}^{b} d z z \mathcal{C}_{\alpha}^{2}=\left.\frac{z^{2}}{2}\left[\mathcal{C}_{\alpha}^{2}(z)-\mathcal{C}_{\alpha+1}(z) \mathcal{C}_{\alpha-1}(z)\right]\right|_{a} ^{b}
$$

In addition, for integer $\alpha, \mathcal{C}_{\alpha}$ satisfies:

$$
\mathcal{C}_{-\alpha}(z)=(-1)^{\alpha} \mathcal{C}_{\alpha}(z)
$$

Furthermore, the following relation holds for all $\alpha$ and $z$ :

$$
J_{\alpha+1}(z) Y_{\alpha}(z)-J_{\alpha}(z) Y_{\alpha+1}(z)=\frac{2}{\pi z} .
$$

We will use these identities frequently throughout the computations to simplify the results.

By inserting the solution eq. (A.1) into the boundary conditions eq. (3.6), we find:

$$
-b_{\alpha, n}=\frac{\widehat{z}_{\mathrm{IR}} J_{\alpha-1}\left(\widehat{z}_{\mathrm{IR}}\right)+(2-\alpha-\lambda) J_{\alpha}\left(\widehat{z}_{\mathrm{IR}}\right)}{\widehat{z}_{\mathrm{IR}} Y_{\alpha-1}\left(\widehat{z}_{\mathrm{IR}}\right)+(2-\alpha-\lambda) Y_{\alpha}\left(\widehat{z}_{\mathrm{IR}}\right)}=\frac{\widehat{z}_{\mathrm{UV}} J_{\alpha-1}\left(\widehat{z}_{\mathrm{UV}}\right)+(2-\alpha-\lambda) J_{\alpha}\left(\widehat{z}_{\mathrm{UV}}\right)}{\widehat{z}_{\mathrm{UV}} Y_{\alpha-1}\left(\widehat{z}_{\mathrm{UV}}\right)+(2-\alpha-\lambda) Y_{\alpha}\left(\widehat{z}_{\mathrm{UV}}\right)} .
$$

From eq. (A.11) we see that the masses $m_{n}$ are determined by

$$
\left[\widehat{z}_{\mathrm{IR}} J_{\alpha-1}\left(\widehat{z}_{\mathrm{IR}}\right)+(2-\alpha-\lambda) J_{\alpha}\left(\widehat{z}_{\mathrm{IR}}\right)\right]+b_{\alpha, n}\left[\widehat{z}_{\mathrm{IR}} Y_{\alpha-1}\left(\widehat{z}_{\mathrm{IR}}\right)+(2-\alpha-\lambda) Y_{\alpha}\left(\widehat{z}_{\mathrm{IR}}\right)\right]=0 .
$$

Using the asymptotic expansions for small arguments, eqs. (A.2) and (A.3), as well as eq. (A.11), we find that (up to $\mathcal{O}(1)$ prefactors)

$$
b_{\alpha, n} \sim \begin{cases}\widehat{z}_{\mathrm{UV}}^{2 \alpha} & \text { for generic } \lambda \\ \alpha(1-\alpha) \widehat{z}_{\mathrm{UV}}^{2 \alpha-2} & \text { for } \lambda=\lambda_{0} .\end{cases}
$$

For $k e^{-k \ell} \ll m_{n} \ll k$ and generic $\alpha$ and $\lambda$, one then finds that the masses $m_{n}$ are determined by the zeros of $J_{\alpha-1}\left(\widehat{z}_{\mathrm{IR}}\right)$. This gives (see e.g. [31]):

$$
m_{n} \simeq\left(n+\frac{\alpha}{2}-\frac{3}{4}\right) \pi k e^{-k \ell} .
$$


As can be seen from the second line of eq. (A.13), the masses are also determined by the zeros of $J_{\alpha-1}\left(\widehat{z}_{\mathrm{IR}}\right)$ for a tuned UV mass term, as long as $\alpha>1$. For $\alpha<1$, the mass spectrum is instead given by the zeros of $Y_{\alpha-1}\left(\widehat{z}_{\mathrm{IR}}\right)$. However, this only leads to a shift in the spectrum.

Next, we want to verify the approximate results for the decay rates, eqs. (3.14), (3.15) and (3.21). Defining $Z_{\alpha}(z) \equiv J_{\alpha}(z)+b_{\alpha, n} Y_{\alpha}(z)$, with $b_{\alpha, n}$ given by eq. (A.11), the boundary conditions can be rewritten as

$$
Z_{\alpha-1}\left(\widehat{z}_{\mathrm{IR}}\right)=-\frac{2-\alpha-\lambda}{\widehat{z}_{\mathrm{IR}}} Z_{\alpha}\left(\widehat{z}_{\mathrm{IR}}\right) \quad \text { and } \quad Z_{\alpha-1}\left(\widehat{z}_{\mathrm{UV}}\right)=-\frac{2-\alpha-\lambda}{\widehat{z}_{\mathrm{UV}}} Z_{\alpha}\left(\widehat{z}_{\mathrm{UV}}\right) .
$$

Furthermore, we have to determine the normalization constant $a_{\alpha, n}$ from eq. (3.9). Using the above identities for Bessel functions and the boundary conditions eq. (A.15), we find

$$
a_{\alpha, n}=\frac{1}{m_{n}} \sqrt{\left(\widehat{z}_{\mathrm{IR}}^{2}+c\right) Z_{\alpha}^{2}\left(\widehat{z}_{\mathrm{IR}}\right)-\left(\widehat{z}_{\mathrm{UV}}^{2}+c\right) Z_{\alpha}^{2}\left(\widehat{z}_{\mathrm{UV}}\right)}
$$

where $c=2 \alpha(2-\alpha-\lambda)+(2-\alpha-\lambda)^{2} \sim \mathcal{O}(1)$ for generic $\lambda$.

From the definition of the coupling constant, eq. (3.3), and our result for the normalization constant, eq. (A.16), we see that we have to compute the ratio of $Z_{\alpha}$ evaluated at the IR brane to $Z_{\alpha}$ evaluated at the UV brane. By repeatedly using the boundary conditions eq. (A.15), as well as eq. (A.11) and the identities given above, this ratio can be simplified to

$$
\frac{Z_{\alpha}\left(\widehat{z}_{\mathrm{IR}}\right)}{Z_{\alpha}\left(\widehat{z}_{\mathrm{UV}}\right)}=\frac{\widehat{z}_{\mathrm{UV}} Y_{\alpha-1}\left(\widehat{z}_{\mathrm{UV}}\right)+(2-\alpha-\lambda) Y_{\alpha}\left(\widehat{z}_{\mathrm{UV}}\right)}{\widehat{z}_{\mathrm{IR}} Y_{\alpha-1}\left(\widehat{z}_{\mathrm{IR}}\right)+(2-\alpha-\lambda) Y_{\alpha}\left(\widehat{z}_{\mathrm{IR}}\right)}
$$

Since we assume that $\widehat{z}_{\mathrm{UV}} \ll 1$, we can use the small argument expansion, eq. (A.3), to simplify the nominator. Furthermore, assuming that $\widehat{z}_{\mathrm{IR}} \gg 1$, we obtain the following approximation (again up to $\mathcal{O}(1)$ prefactors) for the denominator using the mass quantization condition eq. (A.12):

$$
\widehat{z}_{\mathrm{IR}} Y_{\alpha-1}\left(\widehat{z}_{\mathrm{IR}}\right)+(2-\alpha-\lambda) Y_{\alpha}\left(\widehat{z}_{\mathrm{IR}}\right) \sim\left\{\begin{array}{lll}
\sqrt{\widehat{z}_{\mathrm{IR}}} & \text { for generic } \quad \alpha \text { and } \lambda \\
\sqrt{\widehat{z}_{\mathrm{IR}}} & \text { for } \quad \alpha>1 & \text { and } \lambda=\lambda_{0} \\
\sqrt{\widehat{\mathrm{z}}_{\mathrm{IR}}} \widehat{z}_{\mathrm{UV}}^{2-2 \alpha} & \text { for } \quad \alpha<1 & \text { and } \lambda=\lambda_{0} .
\end{array}\right.
$$

Combining the resulting simplified expression for eq. (A.17) with eqs. (3.3) and (A.16), we can estimate the decay rates on dimensional grounds:

$$
\Gamma_{n} \sim \begin{cases}\left(\frac{m_{n}}{k}\right)^{4+2 \alpha} m_{\mathrm{IR}} & \text { for generic } \alpha>0 \text { and } \lambda \\ \left(\frac{m_{n}}{k}\right)^{4} \frac{m_{\mathrm{IR}}}{\ln ^{2}\left(k / m_{n}\right)} & \text { for } \alpha=0 \text { and generic } \lambda \\ \left(\frac{m_{n}}{k}\right)^{2 \alpha} m_{\mathrm{IR}} & \text { for } \alpha>1 \text { and } \lambda=\lambda_{0} \\ \left(\frac{m_{n}}{k}\right)^{4-2 \alpha} m_{\mathrm{IR}} & \text { for } \alpha<1 \text { and } \lambda=\lambda_{0} .\end{cases}
$$


Here, we have replaced $\widehat{z}_{\mathrm{UV}}$ by $m_{n} / k$ and $\widehat{z}_{\mathrm{IR}}$ by $m_{n} / m_{\mathrm{IR}}$. The logarithmic factor for $\alpha=0$ and generic $\lambda$ arises from the logarithm in the expansion of $Y_{0}$ for small argument, eq. (A.3), inserted into eq. (A.17). The decay rates in eq. (A.19) agree with the results of our simplified calculation, eqs. (3.14), (3.15) and (3.21).

Open Access. This article is distributed under the terms of the Creative Commons Attribution Noncommercial License which permits any noncommercial use, distribution, and reproduction in any medium, provided the original author(s) and source are credited.

\section{References}

[1] S.B. Giddings, S. Kachru and J. Polchinski, Hierarchies from fluxes in string compactifications, Phys. Rev. D 66 (2002) 106006 [hep-th/0105097] [SPIRES].

[2] F. Denef and M.R. Douglas, Distributions of flux vacua, JHEP 05 (2004) 072 [hep-th/0404116] [SPIRES].

[3] A. Giryavets, S. Kachru and P.K. Tripathy, On the taxonomy of flux vacua, JHEP 08 (2004) 002 [hep-th/0404243] [SPIRES].

[4] J.P. Conlon and F. Quevedo, On the explicit construction and statistics of Calabi-Yau flux vacua, JHEP 10 (2004) 039 [hep-th/0409215] [SPIRES].

[5] S. Ashok and M.R. Douglas, Counting flux vacua, JHEP 01 (2004) 060 [hep-th/0307049] [SPIRES].

[6] T. Eguchi and Y. Tachikawa, Distribution of flux vacua around singular points in Calabi-Yau moduli space, JHEP 01 (2006) 100 [hep-th/0510061] [SPIRES].

[7] A. Hebecker and J. March-Russell, The ubiquitous throat, Nucl. Phys. B 781 (2007) 99 [hep-th/0607120] [SPIRES].

[8] I.R. Klebanov and M.J. Strassler, Supergravity and a confining gauge theory: duality cascades and $\chi S B$-resolution of naked singularities, JHEP 08 (2000) 052 [hep-th/0007191] [SPIRES].

[9] F. Brummer, A. Hebecker and E. Trincherini, The throat as a Randall-Sundrum model with Goldberger-Wise stabilization, Nucl. Phys. B 738 (2006) 283 [hep-th/0510113] [SPIRES].

[10] S. Kachru, R. Kallosh, A.D. Linde and S.P. Trivedi, De Sitter vacua in string theory, Phys. Rev. D 68 (2003) 046005 [hep-th/0301240] [SPIRES].

[11] S. Kachru et al., Towards inflation in string theory, JCAP 10 (2003) 013 [hep-th/0308055] [SPIRES].

[12] S. Dimopoulos, S. Kachru, N. Kaloper, A.E. Lawrence and E. Silverstein, Small numbers from tunneling between brane throats, Phys. Rev. D 64 (2001) 121702 [hep-th/0104239] [SPIRES].

[13] S. Dimopoulos, S. Kachru, N. Kaloper, A.E. Lawrence and E. Silverstein, Generating small numbers by tunneling in multi-throat compactifications,

Int. J. Mod. Phys. A 19 (2004) 2657 [hep-th/0106128] [SPIRES].

[14] X. Chen and S.H. Henry Tye, Heating in brane inflation and hidden dark matter, JCAP 06 (2006) 011 [hep-th/0602136] [SPIRES]. 
[15] A. Berndsen, J.M. Cline and H. Stoica, Kaluza-Klein relics from warped reheating, Phys. Rev. D 77 (2008) 123522 [arXiv:0710.1299] [SPIRES].

[16] J.F. Dufaux, L. Kofman and M. Peloso, Dangerous angular KK/glueball relics in string theory cosmology, Phys. Rev. D 78 (2008) 023520 [arXiv:0802.2958] [SPIRES].

[17] B.V. Harling and A. Hebecker, Sequestered dark matter, JHEP 05 (2008) 031 [arXiv:0801.4015] [SPIRES].

[18] X. Chen, Decaying hidden dark matter in warped compactification, JCAP 09 (2009) 029 [arXiv : 0902.0008] [SPIRES].

[19] A.R. Frey, R.J. Danos and J.M. Cline, Warped Kaluza-Klein dark matter, JHEP 11 (2009) 102 [arXiv:0908.1387] [SPIRES].

[20] N. Barnaby, C.P. Burgess and J.M. Cline, Warped reheating in brane-antibrane inflation, JCAP 04 (2005) 007 [hep-th/0412040] [SPIRES].

[21] L. Kofman and P. Yi, Reheating the universe after string theory inflation, Phys. Rev. D 72 (2005) 106001 [hep-th/0507257] [SPIRES].

[22] D. Chialva, G. Shiu and B. Underwood, Warped reheating in multi-throat brane inflation, JHEP 01 (2006) 014 [hep-th/0508229] [SPIRES].

[23] A.R. Frey, A. Mazumdar and R.C. Myers, Stringy effects during inflation and reheating, Phys. Rev. D 73 (2006) 026003 [hep-th/0508139] [SPIRES].

[24] S. Panda, M. Sami and I. Thongkool, Reheating the D-brane universe via instant preheating, arXiv:0905.2284 [SPIRES].

[25] L. Randall and R. Sundrum, Out of this world supersymmetry breaking, Nucl. Phys. B 557 (1999) 79 [hep-th/9810155] [SPIRES].

[26] A. Anisimov, M. Dine, M. Graesser and S.D. Thomas, Brane world SUSY breaking, Phys. Rev. D 65 (2002) 105011 [hep-th/0111235] [SPIRES].

[27] A. Anisimov, M. Dine, M. Graesser and S.D. Thomas, Brane world SUSY breaking from string/M theory, JHEP 03 (2002) 036 [hep-th/0201256] [SPIRES].

[28] T. Gregoire, R. Rattazzi, C.A. Scrucca, A. Strumia and E. Trincherini, Gravitational quantum corrections in warped supersymmetric brane worlds, Nucl. Phys. B 720 (2005) 3 [hep-th/0411216] [SPIRES].

[29] T. Gregoire, R. Rattazzi and C.A. Scrucca, D-type supersymmetry breaking and brane-to-brane gravity mediation, Phys. Lett. B 624 (2005) 260 [hep-ph/0505126] [SPIRES].

[30] E. Shuster, Killing spinors and supersymmetry on AdS, Nucl. Phys. B 554 (1999) 198 [hep-th/9902129] [SPIRES].

[31] T. Gherghetta and A. Pomarol, Bulk fields and supersymmetry in a slice of AdS, Nucl. Phys. B 586 (2000) 141 [hep-ph/0003129] [SPIRES].

[32] A. Ceresole, G. Dall'Agata, R. D'Auria and S. Ferrara, Spectrum of type IIB supergravity on $A d S_{5} \times T^{11}$ : Predictions on $N=1$ SCFT's, Phys. Rev. D 61 (2000) 066001 [hep-th/9905226] [SPIRES].

[33] A. Ceresole, G. Dall'Agata and R. D'Auria, KK spectroscopy of type IIB supergravity on $A d S_{5} \times T^{11}, J H E P 11$ (1999) 009 [hep-th/9907216] [SPIRES]. 
[34] A. Ceresole, G. Dall'Agata, R. D'Auria and S. Ferrara, Superconformal field theories from IIB spectroscopy on $A d S_{5} \times T^{11}$, Class. Quant. Grav. 17 (2000) 1017 [hep-th/9910066] [SPIRES].

[35] P. Breitenlohner and D.Z. Freedman, Stability in gauged extended supergravity, Ann. Phys. 144 (1982) 249 [SPIRES].

[36] P. Breitenlohner and D.Z. Freedman, Positive energy in anti-de Sitter backgrounds and gauged extended supergravity, Phys. Lett. B 115 (1982) 197 [SPIRES].

[37] K. Ghoroku and A. Nakamura, Stability of Randall-Sundrum brane-world and tachyonic scalar, Phys. Rev. D 64 (2001) 084028 [hep-th/0103071] [SPIRES].

[38] A. Delgado and M. Redi, Tachyons in a slice of AdS, Phys. Lett. B 562 (2003) 127 [hep-th/0301151] [SPIRES].

[39] D.B. Kaplan, J.-W. Lee, D.T. Son and M.A. Stephanov, Conformality lost, Phys. Rev. D 80 (2009) 125005 [arXiv: 0905.4752] [SPIRES].

[40] M. Toharia, Odd tachyons in compact extra dimensions, arXiv:0803.2503 [SPIRES].

[41] L. Randall and R. Sundrum, A large mass hierarchy from a small extra dimension, Phys. Rev. Lett. 83 (1999) 3370 [hep-ph/9905221] [SPIRES].

[42] W.D. Goldberger and M.B. Wise, Modulus stabilization with bulk fields, Phys. Rev. Lett. 83 (1999) 4922 [hep-ph/9907447] [SPIRES].

[43] H.J. Kim, L.J. Romans and P. van Nieuwenhuizen, The mass spectrum of chiral $N=2$ $D=10$ supergravity on $S^{5}$, Phys. Rev. D 32 (1985) 389 [SPIRES].

[44] L. Randall and R. Sundrum, An alternative to compactification, Phys. Rev. Lett. 83 (1999) 4690 [hep-th/9906064] [SPIRES].

[45] P. Langfelder, On tunnelling in two-throat warped reheating, JHEP 06 (2006) 063 [hep-th/0602296] [SPIRES].

[46] B.V. Harling, A. Hebecker and T. Noguchi, Energy transfer between throats from a $10 D$ perspective, JHEP 11 (2007) 042 [arXiv:0705.3648] [SPIRES].

[47] S. Kuperstein, Meson spectroscopy from holomorphic probes on the warped deformed conifold, JHEP 03 (2005) 014 [hep-th/0411097] [SPIRES].

[48] T. Gherghetta and J. Giedt, Bulk fields in $A d S_{5}$ from probe D7 branes, Phys. Rev. D 74 (2006) 066007 [hep-th/0605212] [SPIRES].

[49] T.S. Levi and P. Ouyang, Mesons and flavor on the conifold, Phys. Rev. D 76 (2007) 105022 [hep-th/0506021] [SPIRES].

[50] H.-Y. Chen, P. Ouyang and G. Shiu, On supersymmetric D7-branes in the warped deformed conifold, JHEP 01 (2010) 028 [arXiv:0807.2428] [SPIRES].

[51] J.M. Maldacena, The large- $N$ limit of superconformal field theories and supergravity, Adv. Theor. Math. Phys. 2 (1998) 231 [Int. J. Theor. Phys. 38 (1999) 1113] [hep-th/9711200] [SPIRES].

[52] O. Aharony, S.S. Gubser, J.M. Maldacena, H. Ooguri and Y. Oz, Large N field theories, string theory and gravity, Phys. Rept. 323 (2000) 183 [hep-th/9905111] [SPIRES].

[53] I.R. Klebanov and E. Witten, AdS/CFT correspondence and symmetry breaking, Nucl. Phys. B 556 (1999) 89 [hep-th/9905104] [SPIRES]. 
[54] S.S. Gubser, I.R. Klebanov and A.M. Polyakov, Gauge theory correlators from non-critical string theory, Phys. Lett. B 428 (1998) 105 [hep-th/9802109] [SPIRES].

[55] E. Witten, Anti-de Sitter space and holography, Adv. Theor. Math. Phys. 2 (1998) 253 [hep-th/9802150] [SPIRES].

[56] M. Pérez-Victoria, Randall-Sundrum models and the regularized AdS/CFT correspondence, JHEP 05 (2001) 064 [hep-th/0105048] [SPIRES].

[57] T. Gherghetta, Warped models and holography, hep-ph/0601213 [SPIRES].

[58] B. Batell and T. Gherghetta, Holographic mixing quantified, Phys. Rev. D 76 (2007) 045017 [arXiv:0706. 0890] [SPIRES].

[59] B. Batell and T. Gherghetta, Warped phenomenology in the holographic basis, Phys. Rev. D 77 (2008) 045002 [arXiv:0710.1838] [SPIRES].

[60] I.R. Klebanov and E. Witten, Superconformal field theory on threebranes at a Calabi-Yau singularity, Nucl. Phys. B 536 (1998) 199 [hep-th/9807080] [SPIRES].

[61] E. Witten, Multi-trace operators, boundary conditions and AdS/CFT correspondence, hep-th/0112258 [SPIRES].

[62] S. Moroz, Below the Breitenlohner-Freedman bound in the nonrelativistic AdS/CFT correspondence, arXiv:0911.4060 [SPIRES].

[63] I.R. Klebanov and N.A. Nekrasov, Gravity duals of fractional branes and logarithmic RG flow, Nucl. Phys. B 574 (2000) 263 [hep-th/9911096] [SPIRES].

[64] I.R. Klebanov and A.A. Tseytlin, Gravity duals of supersymmetric $\mathrm{SU}(N) \times \mathrm{SU}(N+M)$ gauge theories, Nucl. Phys. B 578 (2000) 123 [hep-th/0002159] [SPIRES].

[65] F. Denef, Les Houches lectures on constructing string vacua, arXiv:0803.1194 [SPIRES].

[66] I.R. Klebanov, P. Ouyang and E. Witten, A gravity dual of the chiral anomaly, Phys. Rev. D 65 (2002) 105007 [hep-th/0202056] [SPIRES].

[67] O. Aharony, A note on the holographic interpretation of string theory backgrounds with varying flux, JHEP 03 (2001) 012 [hep-th/0101013] [SPIRES].

[68] S.S. Gubser, C.P. Herzog and I.R. Klebanov, Symmetry breaking and axionic strings in the warped deformed conifold, JHEP 09 (2004) 036 [hep-th/0405282] [SPIRES].

[69] S.S. Gubser, C.P. Herzog and I.R. Klebanov, Variations on the warped deformed conifold, Comptes Rendus Physique 5 (2004) 1031 [hep-th/0409186] [SPIRES].

[70] D.R. Morrison and M.R. Plesser, Non-spherical horizons. I, Adv. Theor. Math. Phys. 3 (1999) 1 [hep-th/9810201] [SPIRES].

[71] A. Butti, M. Graña, R. Minasian, M. Petrini and A. Zaffaroni, The baryonic branch of Klebanov-Strassler solution: a supersymmetric family of $\mathrm{SU}(3)$ structure backgrounds, JHEP 03 (2005) 069 [hep-th/0412187] [SPIRES].

[72] G. Papadopoulos and A.A. Tseytlin, Complex geometry of conifolds and 5-brane wrapped on 2-sphere, Class. Quant. Grav. 18 (2001) 1333 [hep-th/0012034] [SPIRES].

[73] O. DeWolfe, S. Kachru and H.L. Verlinde, The giant inflaton, JHEP 05 (2004) 017 [hep-th/0403123] [SPIRES].

[74] O. Aharony, Y.E. Antebi and M. Berkooz, Open string moduli in KKLT compactifications, Phys. Rev. D 72 (2005) 106009 [hep-th/0508080] [SPIRES]. 
[75] M.K. Benna, A. Dymarsky, I.R. Klebanov and A. Solovyov, On normal modes of a warped throat, JHEP 06 (2008) 070 [arXiv: 0712.4404] [SPIRES].

[76] A. Dymarsky, D. Melnikov and A. Solovyov, I-odd sector of the Klebanov-Strassler theory, JHEP 05 (2009) 105 [arXiv:0810.5666] [SPIRES].

[77] M. Krasnitz, A two point function in a cascading $N=1$ gauge theory from supergravity, hep-th/0011179 [SPIRES].

[78] E. Caceres and R. Hernandez, Glueball masses for the deformed conifold theory, Phys. Lett. B 504 (2001) 64 [hep-th/0011204] [SPIRES].

[79] X. Amador and E. Caceres, Spin two glueball mass and glueball Regge trajectory from supergravity, JHEP 11 (2004) 022 [hep-th/0402061] [SPIRES].

[80] T. Noguchi, M. Yamaguchi and M. Yamashita, Gravitational Kaluza-Klein modes in warped superstring compactification, Phys. Lett. B 636 (2006) 221 [hep-th/0512249] [SPIRES].

[81] M. Berg, M. Haack and W. Mueck, Glueballs vs. gluinoballs: fluctuation spectra in non-AdS/non-CFT, Nucl. Phys. B 789 (2008) 1 [hep-th/0612224] [SPIRES].

[82] A. Dymarsky and D. Melnikov, Gravity multiplet on KS and BB backgrounds, JHEP 05 (2008) 035 [arXiv:0710.4517] [SPIRES].

[83] M.R. Douglas and G. Torroba, Kinetic terms in warped compactifications, JHEP 05 (2009) 013 [arXiv:0805.3700] [SPIRES].

[84] A.R. Frey, G. Torroba, B. Underwood and M.R. Douglas, The universal Kähler modulus in warped compactifications, JHEP 01 (2009) 036 [arXiv:0810.5768] [SPIRES].

[85] N. Barnaby and J.M. Cline, Tachyon defect formation and reheating in brane-antibrane inflation, Int. J. Mod. Phys. A 19 (2004) 5455 [hep-th/0410030] [SPIRES].

[86] N. Barnaby and J.M. Cline, Creating the universe from brane-antibrane annihilation, Phys. Rev. D 70 (2004) 023506 [hep-th/0403223] [SPIRES].

[87] D. Baumann, A. Dymarsky, S. Kachru, I.R. Klebanov and L. McAllister, Holographic systematics of D-brane inflation, JHEP 03 (2009) 093 [arXiv:0808.2811] [SPIRES].

[88] D. Baumann et al., On D3-brane potentials in compactifications with fluxes and wrapped D-branes, JHEP 11 (2006) 031 [hep-th/0607050] [SPIRES].

[89] D. Baumann, A. Dymarsky, I.R. Klebanov, L. McAllister and P.J. Steinhardt, A delicate universe, Phys. Rev. Lett. 99 (2007) 141601 [arXiv:0705.3837] [SPIRES].

[90] D. Baumann, A. Dymarsky, I.R. Klebanov and L. McAllister, Towards an explicit model of D-brane inflation, JCAP 01 (2008) 024 [arXiv:0706.0360] [SPIRES].

[91] C.P. Burgess, J.M. Cline, K. Dasgupta and H. Firouzjahi, Uplifting and inflation with D3 branes, JHEP 03 (2007) 027 [hep-th/0610320] [SPIRES].

[92] H.-Y. Chen, L.-Y. Hung and G. Shiu, Inflation on an open racetrack, JHEP 03 (2009) 083 [arXiv:0901.0267] [SPIRES].

[93] L. Parker, Gravitons from anomalous decay, Int. J. Theor. Phys. 28 (1989) 1163.

[94] R. Delbourgo and D.-S. Liu, Electromagnetic and gravitational decay of the Higgs boson, Austral. J. Phys. 53 (2001) 647 [hep-ph/0004156] [SPIRES].

[95] M. Kawasaki, K. Kohri and N. Sugiyama, MeV-scale reheating temperature and thermalization of neutrino background, Phys. Rev. D 62 (2000) 023506 [astro-ph/0002127] [SPIRES]. 
[96] G.F. Giudice, E.W. Kolb and A. Riotto, Largest temperature of the radiation era and its cosmological implications, Phys. Rev. D 64 (2001) 023508 [hep-ph/0005123] [SPIRES].

[97] S. Hannestad, What is the lowest possible reheating temperature?, Phys. Rev. D 70 (2004) 043506 [astro-ph/0403291] [SPIRES].

[98] R. Kallosh and A.D. Linde, Landscape, the scale of SUSY breaking and inflation, JHEP 12 (2004) 004 [hep-th/0411011] [SPIRES].

[99] J.P. Conlon, R. Kallosh, A.D. Linde and F. Quevedo, Volume modulus inflation and the gravitino mass problem, JCAP 09 (2008) 011 [arXiv:0806.0809] [SPIRES].

[100] G.D. Kribs and I.Z. Rothstein, Bounds on long-lived relics from diffuse gamma ray observations, Phys. Rev. D 55 (1997) 4435 [Erratum ibid. D 56 (1997) 1822] [hep-ph/9610468] [SPIRES].

[101] A. Falkowski, J. Juknevich and J. Shelton, Dark matter through the neutrino portal, arXiv:0908.1790 [SPIRES].

[102] H. Davoudiasl, J.L. Hewett and T.G. Rizzo, Bulk gauge fields in the Randall-Sundrum model, Phys. Lett. B 473 (2000) 43 [hep-ph/9911262] [SPIRES].

[103] D.J.H. Chung, L.L. Everett and H. Davoudiasl, Experimental probes of the Randall-Sundrum infinite extra dimension, Phys. Rev. D 64 (2001) 065002 [hep-ph/0010103] [SPIRES].

[104] M. Abramowitz and I. A. Stegun eds., Handbook of mathematical functions, Dover Publications, New York U.S.A. (1965). 